\title{
Reasoning Between the Lines: A Logic of Relational Propositions
}

\author{
Andrew Potter \\ Computer Science \& Information Systems \\ University of North Alabama
}

APOTTER1@UNA.EDU

\author{
Editor: Manfred Stede
}

Submitted 08/2017; Accepted 12/2018; Published online 01/2019

\begin{abstract}
This paper describes how Rhetorical Structure Theory (RST) and relational propositions can be used to define a method for rendering and analyzing texts as expressions in propositional logic. Relational propositions, the implicit assertions that correspond to RST relations, are defined using standard logical operators and rules of inference. The resulting logical forms are used to construct logical expressions that map to RST tree structures. The resulting expressions show that inference is pervasive within coherent texts. To support reasoning over these expressions, a set of rules for negation is defined. The logical forms and their negation rules can be used to examine the flow of reasoning and the effects of incoherence. Because there is a correspondence between logical coherence and the functional relationships of RST, an RST analysis that cannot pass the test of logic is indicative either of a problematic analysis or of an incoherent text. The result is a method for analyzing the logic implicit within discursive reasoning.
\end{abstract}

Keywords: Rhetorical Structure Theory, RST, relational propositions, propositional logic

\section{Introduction}

An important contribution of Rhetorical Structure Theory (RST) has been its usefulness in describing the organization of text. An analysis created using RST presents the text structurally, identifying the functional relationships between the parts of the text and positioning them hierarchically to reflect their relative status within the overall textual organization. This provides, among other things, a basis for examination of the nature of the coherence of the text, and it has led to diverse studies and applications in discourse analysis, argumentation, theoretical linguistics, psycholinguistics, and computational linguistics (Taboada \& Mann, 2006a).

An area of research that has received little attention has been the interrelationship between RST and propositional logic. Although the relationship between logic and language has been of longstanding interest among philosophers, logicians, linguists, and psychologists, studies specific to RST and propositional logic are not to be found. And yet the characteristics of RST and propositional logic suggest there may be untapped synergies. For both, the object of analysis is a well-defined domain: in RST, the domain is the text under analysis (Mann \& Thompson, 1987b), and in propositional logic, the domain is the universe of discourse, an explicit or implicit boundary within which the analysis is delimited (Boole, 1854). Both involve analysis of relationships among discourse units at the sentential level, and both can be used to discover the sentential organization of discourse. Both are concerned with discursive coherence, and both are useful in the analysis of discursive reasoning.

The purpose of this paper is to examine the use of RST for rendering and analyzing texts as expressions in propositional logic. The primary claim to be developed is that any text analyzable

(C2019 Andrew Potter

This is an open-access article distributed under the terms of a Creative Commons Attribution License (http://creativecommons.org/licenses/by/3.0/). 
using RST can be restated as a Boolean expression. That is, for any RST structure, there is a corresponding Boolean expression consisting of a set of variables representing the discourse elements and connected using logical operators. The logical relations within such a Boolean expression are inferentially interdependent. A set of rules of negation is formulated, and when applied to a text, they facilitate analysis of logical flows from one part to another. This is useful in analyzing rhetorical structures and their logical consequences for the interpretation of text. The result is a method for analyzing the logic implicit within discursive reasoning.

RST is a functional theory of textual coherence. It defines coherence in terms of the relations that occur between parts of the text. The parts of the text may be elementary discourse units, or they may be structures of multiple related units. While there has been considerable debate as to the appropriate set of relations to be used in such an analysis (e.g., Carlson \& Marcu, 2001; Grosz \& Sidner, 1986; Hovy, 1990; Hovy \& Maier, 1993; Knott, Oberlander, O'Donnell, \& Mellish, 2001; Mann \& Thompson, 1987b; Marcu, 2000; Sanders, Spooren, \& Noordman, 1992; Stede, Taboada, \& Das, 2017), there seems to be general agreement that there are identifiable relations and these may be used in describing the nature of coherence in a text. RST further defines coherence using a set of constraints that define the structural characteristics of the text as a whole. These constraints are identified as completeness, connectedness, uniqueness, and adjacency (Mann \& Thompson, 1987b). The application of these constraints enables the analyst to identify the underlying hierarchical structure of the text.

Logic defines coherence as consistency (Kolodny, 2007; Thagard, 1989). To be considered coherent, the universe of discourse must be free of contradiction. The universe of discourse specifies the assertions constituting the universe and their logical interdependencies. The propositions constituting a coherent universe are true, either as elementary propositions asserted as axiomatic, or as inferred propositions, having been deduced either from elementary propositions or from other inferred propositions in accordance with the rules of inference. Propositional logic includes a set of rules of inference, the logical functions that determine which conclusions may be drawn from a given set of premises (Boole, 1854; Russell, 1906). Thus, a coherent universe of discourse may be viewed as an inferential structure of logically related propositions.

These two concepts of coherence, one based on RST and the other on propositional logic, though distinct, have an important similarity: RST represents a text as discourse units connected by relations, and logic represents the universe of discourse as propositions connected by logical operators. If it were the case that RST relations corresponded one to one with logical operators and units with propositions, the task of restating RST analyses as Boolean expressions would not be interesting. However, the task is not so simple. RST relations do not correspond one to one with logical operators. As will be developed in Section 3, teasing out the logical expressions corresponding to RST relations involves a close analysis of the RST definitions as well as examination of examples of RST analyses. Some of the resulting expressions are complex. Of additional concern is the ostensible assumption of parity between elementary discourse units and propositions. In logic, a proposition is defined as being either true or false, but not both, a definition normally associated with declarative sentences. But the discourse units of RST need not be declarative. They can be any sentence type whatsoever, and in some cases, the units are sentence fragments or dependent clauses. While this issue may be addressed in part through the recognition that many non-declarative units may be restated as propositions, this does not fully speak to the issue.

To fully address this issue, it is first necessary to consider propositional logic within the larger context of Boolean algebra. Propositional logic and the Boolean algebra are interchangeable conceptualizations. Essentially, propositional logic is an application of the Boolean algebra. The logical operations of the Boolean algebra correspond to the theorems of propositional logic, and Boolean variables correspond to elementary propositions. Boolean logic is more general and lends 
itself to a variety of applications, including the design of switching circuits, mathematics, set theory, digital logic, and database query languages. Although when discussing propositional logic we tend to speak in terms of truth values and truth functions, these semantics are logically arbitrary, and we could just as well speak of + and,- 1 and 0 , yes and no, satisfiability and unsatisfiability, or any other bivalent conceptualization, such as belief and non-belief, positive and negative regard, desire and indifference, interest and disinterest, understanding and misunderstanding, or ability and inability. To the extent that the primitives of RST can be understood in terms of bivalent values, they are amenable to logical treatment. Any discourse unit that can be either accepted or rejected can be treated as a proposition and subjected to the rules of inference.

Furthermore, in this paper, discourse units are of less concern as isolated units, but rather are of interest primarily with respect to the role they perform within a relational structure. This is perhaps most easily understood in terms of relational propositions, the alter ego of RST relations (Mann \& Thompson, 1986a, 1986b, 2000a). Relational propositions will be explained in detail shortly, but for now, suffice it to say that they are implicit assertions that arise between clauses within a text and are essential to the effective functioning of the text. They are RST relations reformulated as propositions. A relational proposition consists of a predicate and two variables. The predicate corresponds to an RST relation, and the variables map to the units or spans the relation references, one to the nucleus, the other to the satellite. The predicate defines the relationship between these variables. These propositions provide the basis for the logical interpretation of RST analyses. While it is convenient to discuss relational propositions in terms of RST relations, as propositions, these relations may also be treated as truth-functional assertions. Thus, the perspective taken in this paper is that units can be considered not only within a broader Boolean conceptualization, but that their interpretation is reliant on the role they play as arguments within their relational propositions. The value of this approach should become apparent through the analysis of specific relations and structures in Sections 3-5.

An additional potential concern is with text spans. In RST, there are, in addition to units and relations, text spans that represent uninterrupted linear intervals of text, consisting of multiple units and relations. The Mann and Thompson (2000b) analysis shown in Figure 1 contains several such spans, such as the span represented as $2-4$, representing the relations occurring among units 2,3 , and 4. Such multiunit text spans are not explicitly defined in RST, raising the possibility of difficulties in logical interpretation. The ELABORATION relation shown here is between one unit and an EVIDENCE span of two others. Mann and Thompson (2000a) claimed the corresponding relational proposition would be a complex and framework-dependent assertion. However, the only framework upon which the corresponding relational proposition is dependent is Rhetorical Structure Theory itself. The use of multiunit text spans is a diagrammatic shorthand for the structures encapsulated below them, and from the logical perspective, they function much the same as parentheses in a symbolic expression. This will be discussed in detail in Section 2.2.

The organization of the remainder of this paper is as follows. Section 2 presents brief overviews of the theoretical concepts used in the paper. These include RST, relational propositions, and propositional logic. These overviews are followed in Section 3 with an analysis of RST relations, providing a specification of the logical form of each. Section 4 presents a set of rules for negating relational propositions. These rules are based on potential points of failure within the logical forms. In Section 5, the logical forms and the rules of negation are used to examine the logical coherence of two texts. Finally, Section 6 provides a perspective on this research, locates it within the larger context of studies in the relationship between coherence relations and logic, summarizes the results, and suggests some areas for future application and research. 


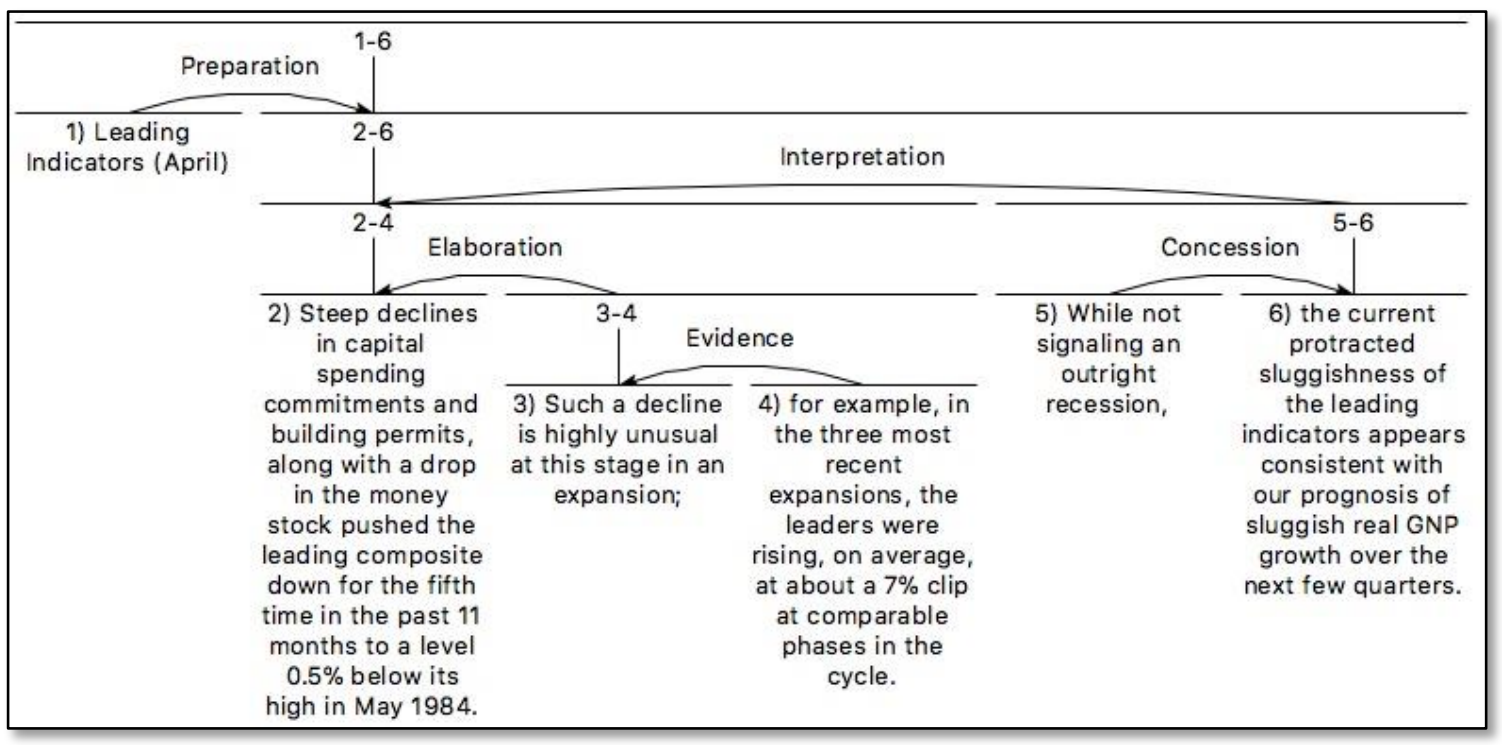

Figure 1. RST analysis containing multiunit spans

\section{Theoretical Background}

This section provides brief overviews of the major theoretical frameworks that support this paper. These include Rhetorical Structure Theory (RST), relational propositions, and propositional logic. RST provides the foundation for describing the sentential organization of a text. It also provides the framework for using relational propositions. The theory of relational propositions is an alternative conceptualization of RST, such that the relationships between text spans are viewed as implicit assertions that occur between clauses in a text. Propositional logic can then be used to define these assertions as logical expressions constructed of propositions and logical operators.

\subsection{Rhetorical Structure Theory}

Rhetorical Structure Theory (RST) is a descriptive theory of text organization. It is a tool for describing and characterizing texts in terms of the relations that hold among the clauses comprising the text. The elements of RST are relations, schemas, schema applications, and structures. An RST relation consists of three parts: the satellite, the nucleus, and the relation. The satellite and nucleus are text spans. The distinction between satellite and nucleus arises as a result of the asymmetry of the relations. Within a relation, the nucleus is more salient than the satellite. Relations are defined in terms of four fields: constraints on the nucleus, constraints on the satellite, constraints on the combination of nucleus and satellite, and the intended effect. For example, in the definition of the EVIDENCE relation, the constraint on the nucleus is that the reader might not believe the information presented in the nucleus, the constraint on the satellite is that the reader must believe it or at least find it credible, and the constraint on the combination of the nucleus and satellite is that by comprehending the satellite, the reader will have an increased belief in the nucleus. The effect of EVIDENCE is that the reader's belief in the nucleus is increased. All of these fields must be satisfied for an application of the EVIDENCE relation.

Mann and Thompson (1987b) proposed that relations could be grouped into two general categories: presentational and subject matter. The distinction is based on intended effect. As defined by Mann and Thompson (p. 18), presentational relations are relations "whose intended effect is to increase some inclination in the reader, such as the desire to act or the degree of positive regard for, belief in, or acceptance of the nucleus." An instance of a presentational relation is, when successful, an act of persuasion. Subject matter relations are relations whose intended effect is that 
the reader recognize the relation in question. Unlike the presentational relations, they involve no act of persuasion. The significance of this distinction for determining logical form will become clear in Section 3.

Schemas are abstract patterns used to specify the relations that may hold among a small set of text spans. Mann and Thompson (1987b) defined five schema types: 1) a relation with a nucleus and satellite, 2) multiple relations with a single nucleus and a satellite for each relation, 3) contrastive multinuclear relations, 4) joint multinuclear relations, and 5) sequential multinuclear relations. The first schema, a relation with a nucleus and a satellite, is employed for most RST relations and is the schema most often found in texts. Schema applications define the conventions for schema instantiation. By convention, the application of schemas places no constraint on the order of spans (i.e. the nucleus may precede or follow the satellite), multi-relational schemas do not restrict which relations are used in their application, and any relation may be repeated multiple times in the application of a schema.

The RST structure of a text refers to its overall composition in terms of schema applications. For the text to be judged coherent, it must conform to four constraints: completeness, connectedness, uniqueness, and adjacency. To be complete, the text must be analyzable as a unified structure. To meet the connectedness constraint, all text spans must be either minimal units or a constituent within a schema application. Uniqueness requires that each schema application consist of a unique set of text spans. The adjacency constraint states that the text spans comprising any schema application constitute one contiguous text span. A consequence of these constraints is that an analysis performed using RST results in a tree structure. RST has been used for a variety of purposes. It has been used as a way of describing the relations between clauses of a text, irrespective of explicit grammatical or lexical signals. It has been used for analysis of a wide range of text types, such as expository prose, narrative discourse, and news broadcasts. It has been used in studies in contrastive rhetoric. Of importance in this paper, RST has been used as a framework for investigating relational propositions, which are the topic of the next section.

\subsection{Relational Propositions}

Relational propositions are implicit coherence-producing assertions that serve to bind together the explicit parts of a text and are essential to the effective functioning of the text. This example (updated for the modern reader) from Mann and Thompson (1986b) is illuminating:

I love to collect classic automobiles. My favorite car is my 1899 Duryea.

I love to collect classic automobiles. My favorite car is my 2008 Toyota Camry.

Even without knowing much about classic cars, most readers would agree that the first text is coherent and the second is not. In the first text, there is an implicit relational proposition that binds the two parts of the text together. The relational proposition asserts that the second part of the text is an elaboration of the first. The reader does not need to be explicitly informed of this relationship. The parts go together. In the second text, this is not the case. The second part of the text does not follow from the first. So essential are relational propositions that their failure may destroy the coherence of a text. These propositions are pervasive throughout the text, and form the basis for various kinds of inference. While lying outside mainstream RST research (Taboada \& Mann, 2006b), the existence and nature of these propositions has been generally acknowledged (Mann \& Thompson, 1986a, 2000a; Nicholas, 1994; Taboada \& Mann, 2006b).

For each relation in an RST analysis, there is a corresponding relational proposition. RST and relational propositions provide parallel accounts of coherence. While RST accounts for coherence relations among the spans within a text, they do not identify the implicit relational acts that account for how the text functions (Mann \& Thompson, 1986b). That is the unique contribution of 
relational propositions. Because relational propositions may be derived from the definitions of RST relations, they need not be sought directly in the text itself (Mann \& Thompson, 1986a, 2000a). This means that relational propositions may be used as adjunct to RST relations.

A relational proposition consists of a predicate and a pair of arguments. The predicate corresponds to the RST relation, and the arguments correspond to the satellite and nucleus. As presented by Mann and Thompson (2000a), the ordering of arguments in a relational proposition need not reflect their original ordering in the text, nor their canonical orderings in RST. The satellite precedes the nucleus, such that $(s)$ predicate $(n)$. For example, the relational proposition for the Duryea example above would be

(My favorite car is my 1899 Duryea) IS AN ELABORATION OF (I love to collect classic automobiles)

These expressions can be made more concise by using a conventional predicate notation with the initial argument as the satellite and the second argument as the nucleus; for example the relational proposition corresponding to the ELABORATION relation would be elaboration $(s, n)$.

For relational propositions to serve as truth-functional representations of RST structures, they not only need to be capable of expressing elementary relational assertions, they must be applicable to complete RST structures. This is an area that has not been previously addressed. As noted above, Mann and Thompson (2000a) claimed that representing multiunit text spans would be a complex framework-dependent undertaking, and did not address it. They confined their research to simple relational propositions. However, RST structures contain multiunit text spans. For example the structure shown above in Figure 1, from (Mann \& Thompson, 2000b), contains several multiunit spans $(1-6,2-6,2-4,3-4$, and 5-6). As noted earlier, such text spans are a diagrammatic shorthand for the structures appearing below them. They provide the same organizational function as parentheses in a symbolic notation. 3-4 is one group of units, 5-6 is another. There is a nesting of relational propositions that corresponds to the structure of the RST tree. The EVIDENCE relation between units 3 and 4 is the satellite of the ELABORATION relation. The CONCESSION relation between units 5 and 6 is the satellite of the INTERPRETATION relation, of which the ELABORATION relation is the nucleus. The resulting structure is the nucleus of the PREPARATION relation:

\section{preparation(1,interpretation(concession(5,6), elaboration(evidence(4,3),2)))}

Thus nested relational propositions enable the identification, not only of individual RST relations as assertions, but the expression of an entire RST structure as a relational proposition. The evaluation of such an expression begins with the innermost relational propositions, and works outward through the nested propositions. This suggests that there is a logical dependence that flows from the leaf nodes to the root. Such an evaluation requires that each relation have a logical specification. Supplying these specifications is the topic of Section 3 of this paper.

\subsection{Propositional logic}

In propositional logic, propositions are treated as primitive units that may be joined using logical operators to form more complex logical expressions. These logical operators are used in this paper:

$\begin{array}{lll}\text { Negation } & \neg & \neg p \\ \text { Conjunction } & \wedge & p \wedge q \\ \text { Disjunction } & \vee & p \vee q \\ \text { Exclusive Disjunction } & \underline{\vee} & p \underline{\vee} q \\ \text { Material Implication } & \rightarrow & p \rightarrow q \\ \text { Material Equivalence } & \leftrightarrow & p \leftrightarrow q \\ & & \end{array}$


When propositions are combined using these operators, the resulting expressions depend on the constituent propositions and the semantics of the operators for their truth value. If $p$ is true then $\neg p$ is false. The conjunction of two propositions $(p \wedge q)$ is true if both $p$ and $q$ are true. Disjunction can be defined in terms of negation and conjunction, as $\neg(\neg p \wedge \neg q))$, i.e., it is not the case that both $p$ and $q$ are false; $(p \vee q)$ is true only if either $p$ or $q$ or both are true. Exclusive disjunction ( $p$ $\underline{\vee} q$ ) is true if $p$ is true or $q$ is true but not both $p$ and $q$ are true. Material implication states that ( $p$ $\rightarrow q$ ) is true if it is the case that $\neg(p \wedge \neg q)$. That is, it will never be the case that $p$ is true when $q$ is false. Material equivalence (or biconditionality) is a relation of mutual implication. If ( $(p \rightarrow q)$ $\wedge(q \rightarrow p))$, then $(p \leftrightarrow q)$.

For logical definitions of RST relations, discourse units within a satellite-nucleus relation are identified using the symbols $s$ and $n$. For example, the definition of ANTITHESIS, discussed below in section 3.1.1, is

$$
((s \vee n) \wedge \neg S) \rightarrow n
$$

For structures involving more than two variables, the traditional symbols from propositional logic are used. Thus, a structure including three discourse units identifies the symbols $p, q$, and $r$, as in

$$
(((p \rightarrow q) \wedge p) \rightarrow q) \wedge(((r \rightarrow q) \wedge r) \rightarrow q)
$$

And when providing a logical interpretation of a specific RST analysis, the units will be identified numerically, reflecting the numbering of units in the analysis, such as

$$
(((1 \rightarrow 2) \wedge 1) \rightarrow 2) \wedge(((3 \rightarrow 2) \wedge 3) \rightarrow 2)
$$

The application of logic to RST, as with any application of logic to the real world, involves the use of judgment under conditions of uncertainty. Mann and Thompson (1987b) specify that when specifying an RST relation between text spans, the analyst must make a judgment with respect to each of these spans, and that owing to the nature of text analysis, these judgments must be made in terms of plausibility rather than certainty. Additional uncertainty will be introduced with the logical interpretations of the relations. The RST relations, although specific, are applicable over a range of situations, and some will conform more closely to the interpretations than others. Subject to these limitations, the following interpretation of RST relations will identify plausible inferential characteristics of rhetorical relations and show how they can be modeled using elementary operations of formal logic.

\section{A Logical Interpretation of Rhetorical Relations}

For RST to be useful in rendering texts as expressions in propositional logic, it is necessary to establish that for each RST relation there is a corresponding logical form. In some cases this amounts to a simple one-to-one correspondence between the relation and a standard rule of inference, and in others the interpretation results in more complex inferential expressions. For each RST relation it is necessary to examine the nature of the asymmetry between the satellite and nucleus. In some cases the relationship is clearly implicative. In others, discovery of the inferential quality of the relation requires more probing. This section presents a discussion of each of the RST relations in the extended Mann and Thompson relation set, as identified on the RST Website, ${ }^{1}$

\footnotetext{
${ }^{1}$ http://www.sfu.ca/rst/index.html
} 
providing a logical interpretation and rationale for each. The relations are grouped according to their traditional categories, starting with presentational relations, followed by subject matter relations and then multinuclear relations.

\subsection{Presentational Relations}

Presentational relations are relations "whose intended effect is to increase some inclination in the reader, such as the desire to act or the degree of positive regard for, belief in, or acceptance of the nucleus" (Mann \& Thompson, 1987b, p. 18). An instance of a presentational relation is, when successful, an act of persuasion. In each of the presentational relations, the satellite serves as the impetus for the reader's acceptance of the intended effect. In this way the writer seeks to influence the reader's reasoning processes. Increases in belief, desire, acceptance, regard, understanding, ability, and interest are the results of inferences specified by the relational propositions associated with these relations. Consequently, these relations are logically argumentative; that is, the logical form of each relation presents a valid argument, consisting of a set of premises and a conclusion which follows from the premises. A consequence of this is that the logical forms of the presentational relations are tautologies. That these relations are tautologous arises due to the relationship between valid arguments and tautologies. For any valid argument, there is a corresponding conditional statement whose antecedent is the conjunction of the premises and whose consequent is the conclusion, and this corresponding statement will be a tautology (Copi, 1967). Thus any relation that is logically argumentative will be tautologous. The implications of this will be explored in detail in Sections 4 and 5.

\subsubsection{ANTITHESIS}

With the ANTITHESIS relation, the intended effect is to increase the reader's positive regard for the situation presented in the nucleus. The satellite is incompatible with the nucleus, such that the reader cannot have positive regard for both the nucleus and the satellite. This incompatibility increases the reader's positive regard for the nucleus. In this example of ANTITHESIS, from Thompson and Mann (1987),

S: Rather than winning them with our arms,

$\mathrm{N}$ : we'd win them by our example, and their desire to follow it.

two alternatives are presented, with the nucleus clearly preferred over the satellite. The logic is simple, based on disjunctive syllogism: $(((s \vee n) \wedge \neg s) \rightarrow n)$. To paraphrase, $s$ or $n$ but not $s$, therefore $n$.

\subsubsection{CONCESSION}

With the CONCESSION relation, the writer concedes the situation presented in the satellite and asserts that, though there might seem to be a potential incompatibility between the satellite and the nucleus, the satellite and nucleus are indeed compatible. The writer holds the nucleus in positive regard, and by indicating a lack of incompatibility with the satellite, the writer seeks to increase the reader's positive regard for the nucleus. To understand the logic of CONCESSION, it is necessary to consider the situation in which it is presented. In their paper on concessive relations, Thompson and Mann (1986, p. 441) observed:

Only in terms of its discourse context can we understand how concession is a 'conceding' of something: it concedes the potential incompatibility of two situations in order to forestall an objection that could interfere with the reader's belief of the point the writer wants to make. 
The objection having been forestalled, its potential interference with the reader's belief has been removed. An example of CONCESSION occurs in the Common Cause letter, as analyzed by Mann and Thompson in several of their papers (Mann \& Thompson, 1986a, 1986b; Thompson \& Mann, 1987):

S: Tempting as it may be,

$\mathrm{N}$ : we shouldn't embrace every popular issue that comes along.

The satellite and nucleus are not incompatible: one may be tempted, yet still resist embracing every popular issue. As such, it is not the case that the satellite provides grounds for rejecting the nucleus:

$$
\neg(s \rightarrow \neg n)
$$

And upon neutralizing this objection, the writer further invites the reader to infer from this the claim presented by the nucleus. If the satellite does not imply the negation of the nucleus, then the nucleus holds. The reasoning thus becomes an instance of modus ponens:

$$
((\neg(s \rightarrow \neg n) \rightarrow n) \wedge \neg(s \rightarrow \neg n)) \rightarrow n
$$

ANTITHESIS and CONCESSION both increase positive regard for the nucleus at the expense of the satellite. ANTITHESIS negates the satellite, and CONCESSION dismisses it. In analyzing texts, it can sometimes be difficult to determine which relation is applicable. In the analysis shown above in Figure 1, Mann and Thompson coded the relation for text span 5-6 as concessive: although there is no recession, the economy is not doing all that well. The antithetical reading is that the writer presents a choice between outright recession and protracted sluggishness, and ruling out recession leads to an inference of protracted sluggishness. The writer is negating the satellite while confirming the nucleus. That fits the logical form of ANTITHESIS precisely. In this manner, the logical forms can be useful in analyzing rhetorical structures. This will be developed further in Section 5.

\subsubsection{EVIDENCE}

With the EVIDENCE relation, the satellite provides evidence in support of the nucleus. For the relation to achieve its intended effect, it is necessary that the reader accept the satellite, either axiomatically as an assumption, or inferentially derived from subordinate relations in the text structure. The reader must recognize the implicative relationship between the satellite and the nucleus, with the result that the reader's belief in the nucleus is increased. In argumentative terms, the satellite is the ground and the nucleus is the claim. The implicative relationship is subject to two constraints. EVIDENCE must be defined to specify that if the antecedent is believable, the consequent will also be believable (material implication) and that the antecedent is believable (modus ponens). Material implication specifies a conditional relationship between the antecedent and the consequent, such that if the antecedent is believable, the consequent will also be believable: $p \rightarrow q$. By definition, material implication does not specify whether the antecedent is true or not. As such, material implication is hypothetical. The EVIDENCE relation is not hypothetical. To achieve its effect, EVIDENCE requires that the antecedent (i.e. the satellite) be asserted. Consequently the implicative relationship is, in addition to material implication, modus ponens:

$$
((s \rightarrow n) \wedge s) \rightarrow n
$$


POTTER

Although the writer anticipates that the reader will accept the satellite, no such assumption is made about the nucleus. If such an assumption could be made, there would be no need for the EVIDENCE relation: the relation could be more properly coded as ELABORATION. In this example of the EVIDENCE relation,

$\mathrm{N}$ : The program as published for calendar year 1980 really works.

S: In only a few minutes, I entered all the figures from my 1980 tax return and got a result which agreed with my hand calculations to the penny.

the writer is praising an income tax preparation software application; efficiency and consistency between programmatic and hand calculations are presented as evidence that the tax program really works (Mann \& Thompson, 1987b). The satellite is asserted and is in an implicative relationship with the nucleus. As discussed below, this use of the modus ponens is applicable to the remaining presentational relations, including JUSTIFY, ENABLEMENT, MOTIVATION, BACKGROUND, PREPARATION, SUMMARY, and RESTATEMENT.

\subsubsection{JUSTIFY}

The satellite of JUSTIFY is used to increase the reader's readiness to accept the writer's right to present what follows in the nucleus. This right to present may come from a variety of sources for use in a variety of situations. For example, the writer may wish to assert a position of authority, make known educational qualifications or professional credentials, announce a leadership role within an organization, or cite qualifying experience within the area under discussion. In this example from AI Magazine, Ken Forbus (2016) makes reference to his experience and expertise in artificial intelligence:

S: Indeed, in my experience,

$\mathrm{N}$ : today's general-purpose AI systems tend to skate a very narrow line between catatonia and attention deficit disorder.

His expertise is invoked to provide authority to the claim. Without this authority, the comparison of AI systems to catatonia and attention deficit disorder would seem unworthy of attention. Writers may also use JUSTIFY when seeking to set aside authority and speak inclusively, so as to obtain acceptance of what follows. A good example of this is the famous quotation from US President John Kennedy, in which he used JUSTIFY to support an ANTITHESIS:

S: And so, my fellow Americans:

$\mathrm{N}$ : ask not what your country can do for you; ask what you can do for your country.

Assuming an authoritative stance when asking the audience to give up their selfish desires in favor of altruistic contributions would be to risk accusation of self-serving hypocrisy. JUSTIFY is also used when the writer is in a subordinate position, seeking permission to speak, or as a (perhaps ritualistic) show of respect, as when a lawyer in a U.S. courtroom begins an oral argument with the phrase, May it please the Court.... Stede et al. (2017) observe that with JUSTIFY the satellite may be used to present a basic attitude of the writer, making it easier for the reader to accept the nucleus. An example of this can be found in the Common Cause analysis, as shown in Figure 2. The writer seeks to dispel any perception of conflict between his personal views and his strategic view regarding the nuclear freeze proposal. JUSTIFY achieves credibility through advantageously positioning the writer with respect to the claim made in the nucleus. 


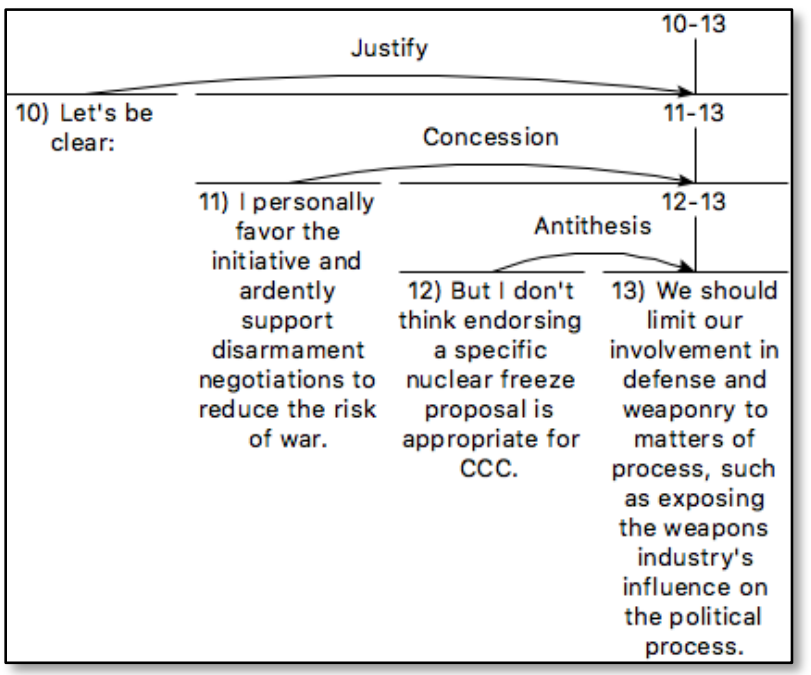

Figure 2. Common Cause example of JUSTIFY

\subsubsection{Motivation and ENABLEMENT}

Mann and Thompson (1987b) treat MotiVATION and ENABLEMENT as a subgroup within the presentational relations. In both of these relations the nucleus presents an action. The action may be presented as an imperative or as an implicit call to action. MOTIVATION increases the reader's desire to perform the action, and ENABLEMENT informs the reader how to do it. These two relations are often found together, their satellites linked to a common nucleus. This structural arrangement has sometimes been referred to as a MOTIVATION-ENABLEMENT schema (Mann \& Thompson, 1987b). The relations work well together, where one increases a desire and the other shows how to satisfy it.

Motivation and ENABLEMENT are implicative and follow the same logical form as EVIDENCE. If the MOTIVATION satellite fails to motivate or if the ENABLEMENT satellite fails to increase the reader's ability to perform the action, the action presented in the nucleus is less likely to occur. When multiple satellites of a presentational relation share the same nucleus, they combine conjunctively to support the nucleus. In the MOTIVATION-ENABLEMENT schema, desire and ability join forces to persuade the reader to take the indicated action.

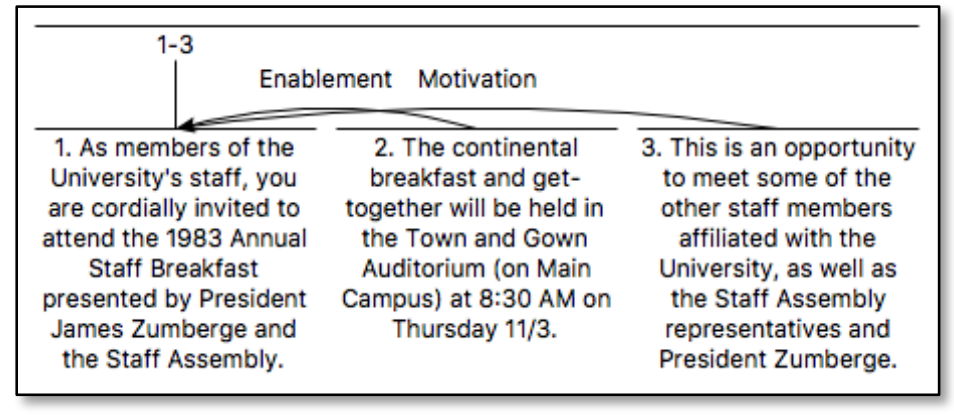

Figure 3. Linked argument using MOTIVATION and ENABLEMENT

In the analysis shown in Figure 3, from Mann and Thompson (1987a), the action to be taken is to attend the 1983 Annual Staff Breakfast. Information about when and where the event will be held enables the reader to attend, and the opportunity to meet other staff members, staff assembly 
representatives, and the university president provides reason for attending. The absence of either satellite would not necessarily lead to failure to take action. But given the presence of both satellites, if either of the relational propositions were to fail, i.e. if the intended motivation were demotivating, or the enablement contained incorrect or irrelevant information, then the MotiVATION-ENABLEMENT schema would fail. Taken together they present a linked argument, sharing a common locus of effect:

$$
(((2 \rightarrow 1) \wedge 2) \rightarrow 1) \wedge(((3 \rightarrow 1) \wedge 3) \rightarrow 1)
$$

\subsubsection{BACKGROUND}

BACKGROUND is used to assure comprehensibility of the text. A nucleus that would otherwise be incomprehensible is rendered understandable by virtue of its satellite. In this example from Mann and Thompson (1987a), the satellite provides BACKGROUND for the nucleus. Without the satellite, the nucleus would be difficult and perhaps impossible to understand:

S: I am having my car repaired in Santa Monica (1522 Lincoln Blvd.) this Thursday 19th. $\mathrm{N}$ : Would anyone be able to bring me to ISI from there in the morning or drop me back there by 5 pm please?

BACKGROUND has the same logical form as EVIDENCE, though the implication is one of comprehension rather than belief or acceptance.

\subsubsection{Preparation}

Stede et al. (2017) specified that PREPARATION be used when the satellite serves no stronger purpose than setting the topic for the nucleus. But setting the topic can be decisive in determining whether the writer achieves the intended effect of the text. PREPARATION may determine whether the reader bothers to read the text. In the analysis shown in Figure 4, the title serves to announce and delimit the topic of the text. The satellite precedes the nucleus and tends to make the reader more ready, interested or oriented for reading the nucleus. Used in this way, PREPARATION is intended to increase an inclination in the reader, namely an inclination to read the article. Thus, PREPARATION is presentational and subject to the same logical form as EVIDENCE.

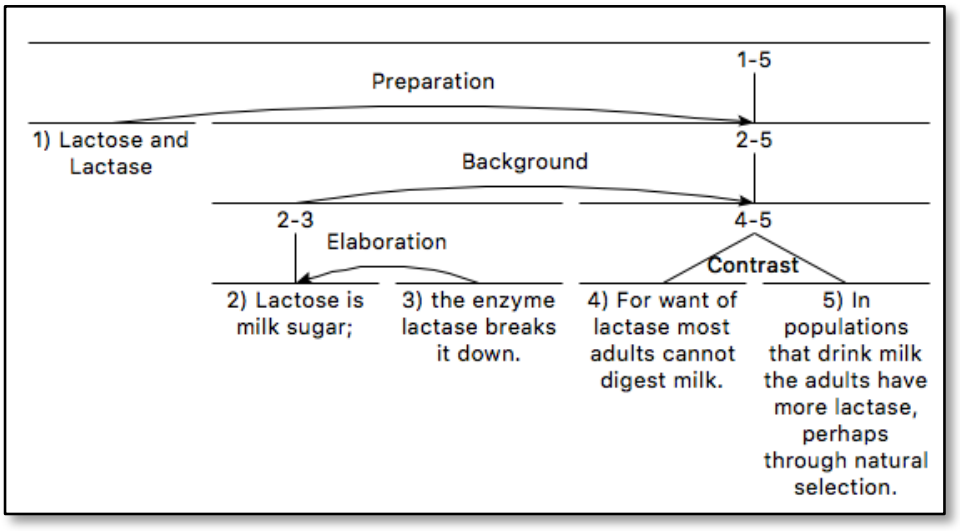

Figure 4. The PREPARATION relation 


\subsubsection{RESTATEMENT and SUMMARY}

The status of RESTATEMENT and SUMMARY as presentational relations is less than obvious. They were originally defined as subject matter relations (Mann \& Thompson, 1987b, 1988). Subsequently, on the RST Website, they were re-categorized as presentational, but their definitions were not updated to reflect this change. With the RESTATEMENT relation, the satellite restates the nucleus, and the satellite and nucleus are of comparable length. The SUMMARY relation is similar. The satellite restates the nucleus, but unlike RESTATEMENT, the satellite is shorter than the nucleus. The defined effect of both of these relations is that the reader recognizes that the satellite restates the nucleus. If this were all there was to say on the matter, it would be difficult to see the intended effect as presentational - there would be no identifiable inclination in the reader that these relations increase. Stede et al. (2017) argued that these relations should be treated as textual, and as with PREPARATION, there is clearly a textual dimension to their use. However, going beyond the definitions, examining examples indicates that they are presentational. RESTATEMENT and SUMMARY are used to reinforce a point the writer seeks to make. In the following example of RESTATEMENT, from Mann and Thompson (1987b), the writer seeks to emphasize the claim that a clean car makes a statement about the character of the owner, and implicitly, that an unkempt car does too:

\section{N: A WELL-GROOMED CAR REFLECTS ITS OWNER}

$\mathrm{S}$ : The car you drive says a lot about you.

This is not simply a case of organizing the text. The RESTATEMENT drives home the point. SUMMARY can be used in the same way. By recapping a complex text with a short summary, the writer can reinforce the claim. Used in this way, RESTATEMENT and SUMMARY support reader comprehension, belief, or action in a manner similar to BACKGROUND and PREPARATION, and thus they adopt the same logical form.

\subsection{Subject Matter Relations}

Subject matter relations are relations whose intended effect is that the reader will recognize the relation in question (Mann \& Thompson, 1987b). Unlike the presentational relations, they involve no act of persuasion. The locus of effect resides in both the nucleus and the satellite. Because the acceptability of the nucleus and the satellite is not in question, the minimal logical form for a subject matter relation could be considered to be the conjunction of the satellite and nucleus, $s \wedge n$. But the asymmetry of the subject matter relations indicate logical information beyond conjunction. The intended effect is a recognition of some feature of the organization of the subject matter (Mann \& Matthiessen, 1990), and the logical forms corresponding to the subject matter relations should capture such features. For example, in a causal relation, the dependency between cause and effect may be represented as an implicative relationship. However, unlike the presentational relations, the point here is not that the implication is needed to establish the acceptability of the consequent. The antecedent and the consequent are both presumed to hold, and the relation indicates that they are in an implicative relationship. Such a relation would take the form, $s \wedge n \wedge(s \rightarrow n)$. From a purely logical perspective, the implicative portion of this form is redundant: $s \rightarrow n$ will follow, by definition, from $s \wedge n$. But the implicative characterizes the inferential processes implicit within the intended effect. The relational proposition corresponding to the logical form of an implicative subject matter relation is true only when both $s$ and $n$ are true and the implication holds. Denial of the implication would be an indication of a failure to achieve the writer's intended effect. In other words, the text would be logically incoherent. This is explored in detail in Sections 4 and 5. The following sections provide definitions of the logical forms for each of the subject matter relations. 


\subsubsection{Causal Relations}

The term cause can be defined broadly as any situation or activity that brings about a change in some other situation or activity. In that sense, a causal relation is a relation that holds between parts of text, in which one part presents a cause and another part the effect. From this perspective, there are several subject matter relations that describe cause and effect relationships. Mann and Thompson (1987b) originally identified the causal relations as consisting of VOLITIONAL CAUSE, Non-Volitional CaUSE, Volitional Result, Non-Volitional Result, and PuRPOSE. They used the concept of volitionality as a means for distinguishing causes that involve the action of an agent, typically but not necessarily an action of a person, from causes that involve consequentiality without a chosen outcome. Some analysts have elected to disregard this distinction between volitionality and non-volitionality. Nicholas (1994) argued that since volitionality modifies only the nucleus or the satellite, but not the relationship between the two, it is rhetorically irrelevant. In their RST annotation guidelines, Stede et al. (2017) ignored volitionality altogether and subsumed the Mann and Thompson relations under the more general categories of CAUSE and RESULT. Because volitionality has no bearing on the logical analysis, I have followed their practice in this discussion of causal relations.

Stede et al. (2017) also specified that the RST relations CONDITION, OTHERWISE, and UNLESS relations should also be treated as causal. These relations, along with PURPOSE, apply to hypothetical situations. With CONDITION, the satellite presents a hypothetical, future, or otherwise unrealized situation upon which the nucleus depends. With OTHERWISE, realization of the nuclear situation prevents realization of the satellite situation. With the UNLESS relation, realization of the nucleus occurs provided that realization of the satellite does not. With PURPOSE, the nucleus is an activity that will lead to the situation identified in the satellite.

Two additional relations that can be considered as causal are MEANS and UNCONDITIONAL. MEANS specifies that the satellite presents a method or instrument which tends to make realization of the activity presented in the nucleus more likely. In this regard it is akin to ENABLEMENT, except the presented activity is not intended to increase an inclination in the reader. The satellite presents a situation that brings about the activity presented in the nucleus. The UNCONDITIONAL relation asserts that the nucleus has no dependency on the satellite. It is a relation of negative causality that is, UNCONDITIONAL negates any causal relation between the satellite and the nucleus. The following provides logical interpretations for each of these causal relations.

\subsubsection{CAUSE and RESUlT}

CAUSE specifies that the satellite caused the nucleus, and RESULT specifies that the nucleus caused the satellite. They are both subject matter implicative. Some activity or situation arises as a result of some other activity or situation. Their relational propositions assert that there is a causal relationship between the satellite and nucleus. Here is an example of CAUSE excerpted from the Salvage and Archaeology analysis (available on the RST Website):

S: Since the objects in a [ship]wreck represent a single moment in time,

$\mathrm{N}$ : they provide better chronological information than even the most carefully excavated terrestrial site.

If the analyst had viewed this relation as argumentative, if there were some doubt that objects in a shipwreck provide better chronological information, then the relation would have been coded as EVIDENCE. But in the analyst's judgment, this is not the case. The intended effect is that the reader recognizes that the satellite is the cause for the nucleus. Implication is needed to establish not the truth of the consequent, but that the antecedent and the consequent are in an implicative relationship. The logical form of the CAUSE relation documents this implicative relationship: 


$$
s \wedge n \wedge(s \rightarrow n)
$$

The RESULT relation is similar, except now the nucleus is the cause: $s \wedge n \wedge(n \rightarrow s)$. In this example from Taboada and Renkema (2008), the speaker's difficulty in smiling at Jesus arises as a result of the demands he places on her. Hence the satellite follows from the nucleus:

S: ... I find it hard sometimes to smile at Jesus.

$\mathrm{N}$ : He can be very demanding.

\subsubsection{MEANS}

The MEANS relation specifies that the satellite presents a method or instrument which tends to make realization of the activity presented in the nucleus more likely. In this analysis from Taboada (2006), MEANS represents features of a method reflected in the design of the human body:

$\mathrm{N}:$... the visual system resolves confusion

S: by applying some tricks that reflect a built-in knowledge of properties of the physical world.

Thus, MEANS is a special case of CAUSE, and it has the same subject matter implicative form.

\subsubsection{CONDITION}

With CONDITION, realization of the satellite results in realization of the nucleus. Because the realization of the nucleus depends upon realization of the satellite, without realization of the satellite there will be no realization of the nucleus. In this example from Mann and Thompson (1987b), the satellite specifies the conditions under which employees are urged to complete new beneficiary designation forms:

$\mathrm{N}$ : Employees are urged to complete new beneficiary designation forms for retirement or life insurance benefits

$\mathrm{S}$ : whenever there is a change in marital or family status.

A change in marital or family status is the condition under which employees are urged to complete new beneficiary designation forms. The reader recognizes that the realization of the nucleus depends on the realization of satellite. As long as the satellite remains unrealized, so will the nucleus. Thus the relation is one of material equivalence $(s \leftrightarrow n)$.

\subsubsection{PuRpose}

PURPOSE is logically similar to CONDITION. With the PURPOSE relation the situation presented in the satellite is to be realized through the activity presented in the nucleus. The satellite is an as yet unrealized result of the nucleus. In this example from Mann and Thompson (1987b),

S: To see which Syncom diskette will replace the ones you're using now

$\mathrm{N}$ : send for our free "Flexi-Finder" selection guide and the name of the supplier nearest you.

The purpose of sending for the selection guide is to determine the appropriate Syncom diskette. Without the activity identified in the nucleus, the situation identified in the satellite is without 
purpose and will not be realized. The logic of PURPOSE is not only $n \rightarrow s$, but also $\neg n \rightarrow \neg s$. So the logic is material equivalence, $n \leftrightarrow s$.

\subsubsection{UNCONDITIONAL}

UNCONDITIONAL has received little attention in the literature, and some studies suggest that it occurs infrequently (e.g. Iruskieta, 2014; Rubin \& Vashchilko, 2012). This may be due in part to difficulties in differentiating it from CONCESSION. Like CONCESSION, although the satellite could conceivably affect the realization of the nucleus, the nucleus is not dependent on the satellite. Figure 5 shows an example from an article on pre-performance anxiety (Brooks, 2013). The nucleus holds, regardless of the value of the satellite: $(s \vee \neg S) \rightarrow n$.

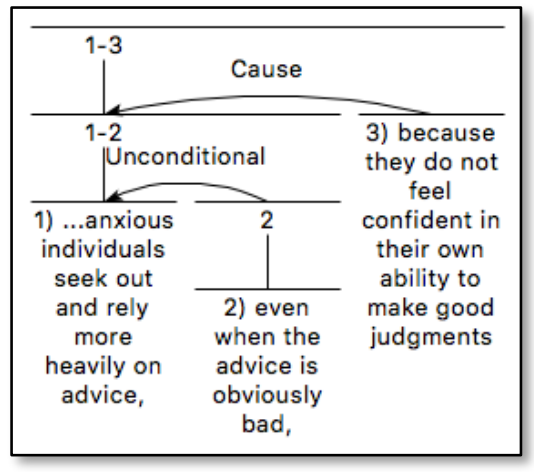

Figure 5. UNCONDITIONAL relation

\subsubsection{OTHERWISE and UNLESS}

The OTHERWISE and UNLESS relations are similar to one another. With OTHERWISE, realization of the nuclear situation prevents realization of the satellite situation. In preventing realization of the satellite situation, the nucleus effectively negates it. In this example from the RST Website,

N: Project leaders should submit their entries for the revised brochure immediately.

S: Otherwise the existing entry will be used.

Only by submitting their new entries can project leaders prevent the use of existing entries, and failure to submit will affirm the satellite. Consequently, OTHERWISE indicates an exclusive disjunction, i.e., $(n \underline{\vee} s)$, or $((n \vee s) \wedge \neg(n \wedge s))$. Either $n$ will hold, or $s$ will hold, but not both. UNLESS is similar to OTHERWISE, except now the realization of the satellite would prevent realization of the nucleus, rather than the nucleus preventing the satellite.

\subsubsection{SOLUTIONHOOD}

With SOLUTIONHOOD, the satellite poses a problem, for which the nucleus presents a solution. The satellite may be presented as a question, request, problem, or other expressed need. The logic of SOLUTIONHOOD is not to be found in the potentially interrogative nature of the satellite. The logic can be derived from its RST definition and corresponding relational proposition. To explain this, it is useful to examine an analogy between interrogatives and propositional functions, proposed by Hintikka (2007). A propositional function is an "expression containing an undetermined constituent," as defined by Whitehead and Russell (1910, p. 92). Because of this undetermined constituent (or free variable), a propositional function is not itself a proposition, so it has no truth value and cannot serve as an element within an argument. A propositional function defines the form of a proposition without asserting the proposition itself. The propositional function $\phi x$ 
specifies some predicate $\phi$ of $x$ without indicating whether there might be any $x$. In this sense, according to Hintikka, a propositional function is a specification of a query. But on the basis of such a query alone, it cannot be known whether any result will be forthcoming. The query could return a null set. The query can be made more specific by binding the variable $x$. This binding, or existential quantification, is expressed as $(\exists x) \phi x$, which asserts that for at least one instance of $x$, $\phi x$ is the case, although the identity of $x$ is unspecified. As such it is a proposition. But to specify that there is an identifiable $x$ for $\phi x$, the variable $x$ must be replaced with a constant, $v$. Since we know that $(\exists x) \phi x$, we can infer that there is some constant that will support this. This is accomplished using existential instantiation:

$$
\begin{aligned}
& (\exists x) \phi x \\
& \therefore \phi v
\end{aligned}
$$

From the query $(\exists x) \phi x$, the answer, $\phi v$ is inferred. With SOLUTIONHOOD, the writer has committed to providing a solution. The problem is not simply posed and left for the reader to resolve. The problem presented by the satellite is an indispensable part of a relation that must be satisfied by the solution presented as the nucleus. The presentment of the problem, whether interrogative or otherwise, obliges the writer to provide an answer. Otherwise there is no SOLUTIONHOOD. The questions, requests, or problems posed in a SOLUTIONHOOD relation are not to be taken out of context. The writer is providing a problem-solution matching set.

The satellite is the specification of a query. Since the writer is committed to providing a solution, the satellite is existentially quantified. The proposition $(\exists x) \phi x$ represents the satellite of the SOLUTIONHOOD relation, where $\phi$ is the problem, and $x$ is the undetermined solution. That is, it is asserted that there is a solution to the problem, but as yet the solution has not been identified. The solution is identified in the nucleus, where $x$ becomes $v$, thanks to existential instantiation. Therefore, SOLUTIONHOOD is an inferential relation between the satellite and the nucleus. Since $(\exists x) \phi x$ and $\phi v$ are propositions, the inference may be restated propositionally as

$$
s \wedge(s \rightarrow n)
$$

Given the assertion of $s, n$. This is not to claim that the answer to a question can be deduced from the question. Rather that, in a coherent text, the posing of a problem can be understood to anticipate the solution, much as a database query uses a subset of first order logic to implicate the result set (Sowa, 2000). An example will make this more concrete. This example is from Mann and Thompson (1985):

S: I'm hungry.

N: Let's go to the Fuji Gardens.

The problem is that the speaker is hungry, and the solution is that going to Fuji Gardens (presumably a restaurant) solves the hunger problem. This relation makes some presuppositions about hunger and the means by which the discomfort that attends it may be relieved. It is the desire to relieve the discomfort associated with hunger that gives the first statement its status as a problem, and it is the relief from discomfort resulting from the act of eating that gives the second statement its status as the solution to the problem. It is through recognition of these presuppositions that the statements achieve coherence, and it is on this level that the inferential nature of the SOLUTIONHOOD relational proposition becomes apparent. Expressed as a propositional function, the problem to be solved is some as yet unidentified source of relief $(x)$. Posed as the satellite to a 
SOLUTIONHOOD relation, the problem is existentially quantified, expressed as $(\exists x) \operatorname{relief}(x)$. The nucleus, using existential instantiation then substitutes Fuji Gardens $(v)$ as the source of relief:

$$
(\exists x) \operatorname{relief}(x) \rightarrow \operatorname{relief}(\text { FujiGardens })
$$

This is not the only effort to define SOLUTIONHOOD in terms amenable to inferential interpretation. Abelen, Redeker, and Thompson (1993) treat Solutionhood as an interpersonal relation, which they define as a relation used for persuasion, and they categorized it alongside presentational relations such as EvidENCE, JUSTIFY, and Motivation. Similarly, Azar (1999) cited the persuasive power of SOLUTIONHOOD in his study of the rhetorical structure of argumentative texts. When leveraging the satellite to accept the nucleus, there would be an inferential dimension in SOLUTIONHOOD. And while persuasiveness may not be a factor in the Fuji Gardens example, it can be found in other cases, such as the Syncom example used by Mann and Thompson (1987b), as well in Azar's analysis. But persuasiveness is not an essential feature of SOLUTIONHOOD, and for the purpose of discovering its underlying logic, it is unnecessary, as has been demonstrated here.

\subsubsection{ELABORATION}

With ELABORATION, the satellite presents additional detail about the situation or some element of subject matter which is presented or inferentially accessible in the nucleus. Mann and Thompson (1987b) identified six subtypes of the relation, as listed in Table 1. As noted by Taboada and Mann (2006b), any one of these subtypes is sufficient for the ELABORATION relation to hold, and in theory it could be possible to treat them as separate relations. And while Carlson and Marcu (2001) did just that, for the purpose of logical interpretation this is unnecessary. The common characteristic among the subtypes is that, as defined, the satellite presents additional detail about the nucleus.

\begin{tabular}{|l|l|}
\hline Nucleus & Satellite \\
\hline Set & Member \\
\hline Abstraction & Instance \\
\hline Whole & Part \\
\hline Process & Step \\
\hline Object & Attribute \\
\hline Generalization & Specific \\
\hline
\end{tabular}

Table 1. ElabORATION subtypes

Whether through set membership, instantiation, mereology, process decomposition, object attribution, or specification, the satellite is logically implicit in the nucleus. Were this subsumptive relationship inaccessible to the reader, the relevance of the satellite to the nucleus would be lost: the inference is necessary for the reader to recognize that the situation presented in the satellite provides additional information for the nucleus. In the example shown in Figure 6, in segment 1 , the writer identifies an ongoing activity. As analyzed by Mann and Thompson (1987a), segment 2 elaborates this by identifying a type of activity (set-member), and unit 3 further details this activity (generalization-specific). Each of the nuclei progressively circumscribe the satellites of their relations. Thus ELABORATION presents a subject matter implication, with the nucleus as antecedent and the satellite as consequent: $s \wedge n \wedge(n \rightarrow s)$. 


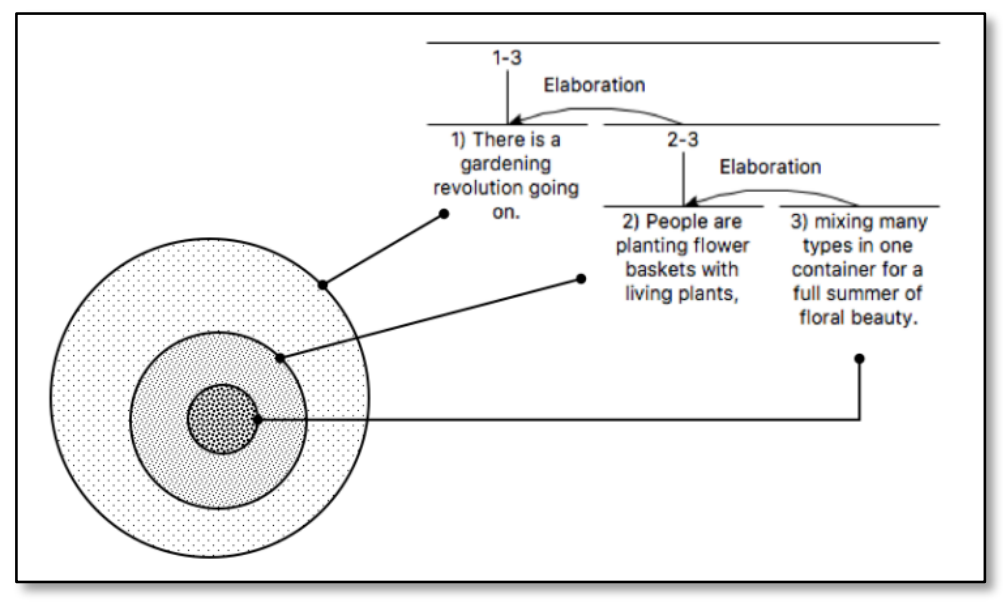

Figure 6. ELABORATION nuclei circumscribe their respective satellites

\subsubsection{EVALUATION and INTERPRETATION}

EVALUATION and INTERPRETATION involve assessment of the situation presented in the nucleus. EVALUATION assesses the situation presented by the nucleus with respect to the writer's positive regard. INTERPRETATION relates the nuclear situation to a framework of ideas not involved in the nucleus and not concerned with the writer's positive regard (Mann \& Thompson, 1987b). In both relations, the assessment presented in the satellite is a plausible inference made on the basis of the information provided in the nucleus. To the extent that the inference offered by the satellite is plausible, the relation is implicative: $((n \rightarrow s) \wedge n) \rightarrow s$. The implicative nature of these relations has been hinted at by several other researchers. Both Stent (2000) and Stede (2008) note that EVALUATION involves the subjective judgment of the writer, and earlier Abelen et al. (1993) categorized both EVALUATION and INTERPRETATION as interpersonal relations. Deriving their definition of the term interpersonal from Halliday and Hasan (1976), Abelen et al. view these relations as being notable for their persuasive power. What distinguishes EVALUATION from the presentational relations is that, while the writer presents an expression of positive regard, the effect is not explicitly directed towards any prospective inclination in the reader. The RST definition of the relation obscures this characteristic, because the effect occurs not in the nucleus but in the satellite. In an excerpt from Mann and Thompson's (1987b) Syncom analysis, shown in Figure 7, the assessment presented in the satellite follows as a result of the information presented in the nucleus. An evaluation that did not follow from its nucleus would be incoherent. Although INTERPRETATION does not deal in positive regard, the logical interpretation is identical to EVALUATION. Unless the interpretation follows from its basis, it will be incoherent. Just as with EVALUATION, the assessment presented in the satellite must be a plausible inference made on the basis of the information provided in the nucleus.

\subsubsection{Circumstance}

The satellite of the CIRCUMSTANCE relation sets the framework within which the reader is intended to interpret the nucleus. In this respect, CIRCUMSTANCE is similar to BACKGROUND, as noted by Stede et al. (2017). The framework imposed by the satellite functions as a constraint on the nucleus, delimiting it with respect to temporal, spatial, or other considerations. Figure 8 shows an analysis with two CIRCUMSTANCE relations, one spatial, the other temporal. Sometimes an instance of CIRCUMSTANCE includes both spatial and temporal constraints, as in the example from the RST Website shown in Figure 9. This serves to illustrate the constraining effect of the relation. 


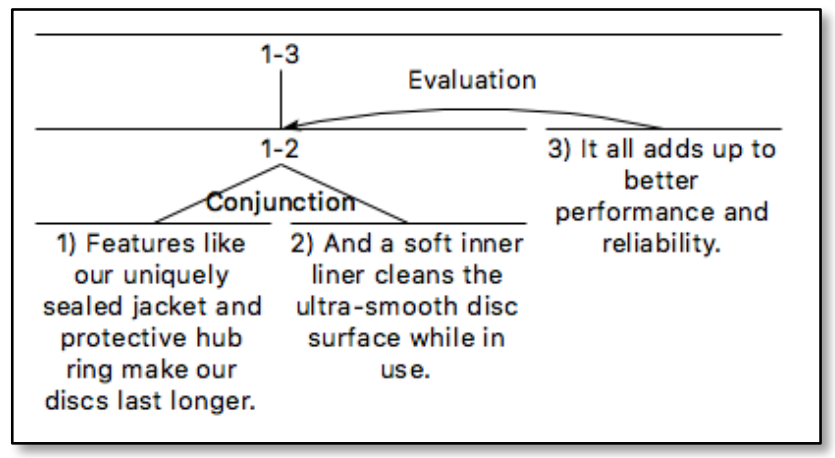

Figure 7. The Evaluation relation

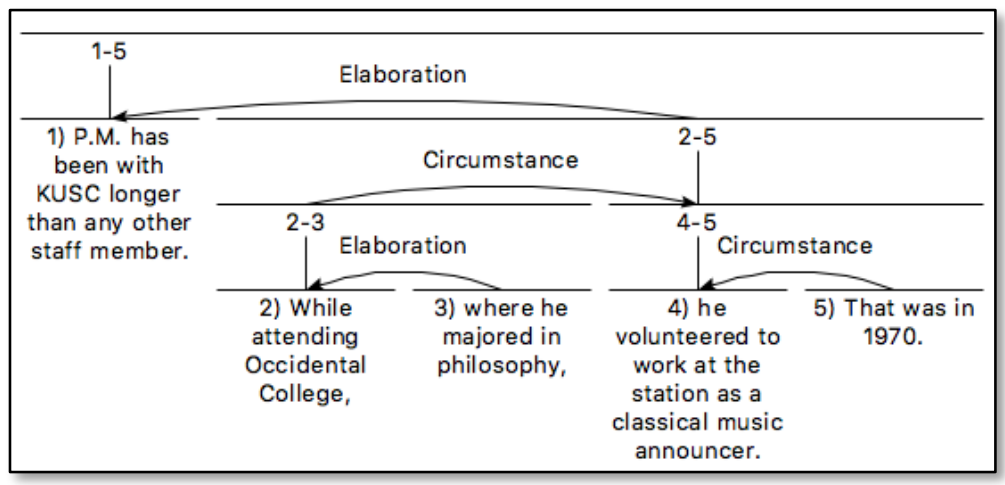

Figure 8. Two CiRCUMSTANCE relations, one spatial the other temporal

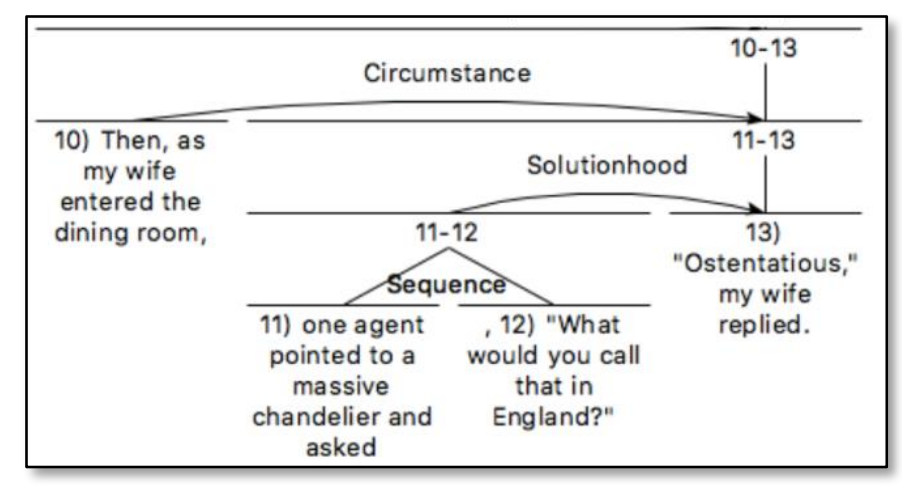

Figure 9. One CIRCUMSTANCE relation with both temporal and spatial characteristics

Although some researchers have found CIRCUMSTANCE to be non-causal (e.g., Carlson \& Marcu, 2001), there are instances that combine temporal or spatial constraints with causality. The following examples use causal CIRCUMSTANCE, the first from Mann and Thompson (1987b) and the second from Stede et al. (2017). In the Visitors Fever example, the writer attributes the cause of the ailment to a visit with relatives in the Midwest. In the second example, deregulation of the electricity market provides the reason for squeezing suppliers: 
N: Probably the most extreme case of Visitors Fever I have ever witnessed was a few summers ago

S: when I visited relatives in the Midwest.

S: When Veag came under pressure because of the deregulation of the electricity market, $\mathrm{N}$ : they compensated for this by squeezing their suppliers.

While CirCumstance is similar to ElABORATION with respect to the satellite providing additional information about the nucleus, the nature of the additional information is quite different. Rather than the nucleus subsuming the satellite, the effect of the additional information provided by the satellite circumscribes the scope of the nucleus. The nucleus holds within the domain specified by the satellite. The satellite specifies a condition under which the nucleus holds. The logical form of CIRCUMSTANCE is subject matter implicative: $s \wedge n \wedge(s \rightarrow n)$.

\subsection{Multinuclear Relations}

Most of the multinuclear relations are logical conjunctions, with exceptions being DISJUNCTION and Multinuclear-Restatement. Disjunction, as the name suggests, is disjunctive. The elements of MULTINUCLEAR-RESTATEMENT are materially equivalent assertions. While the conjunctive nature of CONJUNCTION, CONTRAST, JOINT, and LIST should seem readily apparent, SEQUENCE might seem serially inferential. With SEQUENCE, a succession relationship between the situations is presented. Mann and Thompson suggest recipes as good examples. There is a serial dependency among the nuclei. The dependency could be temporal, process, or any other series. To the extent that this is the case, failure of any nucleus may render the successors unreachable. But this means that for a sequence to hold, all of its members must hold. Hence SEQUENCE may be defined as the logical conjunction of its members.

Mann and Thompson (1985) claimed that relational propositions are always present in multisentence texts. And yet none of their work on the topic mentions multinuclear relational propositions. I propose that they would take the form of $\operatorname{LIST}(n 1, n 2, n 3 \ldots)$, which would transform into the logical expression $n 1 \wedge n 2 \wedge n 3 \ldots$ And DISJUNCTION would follow the same pattern, using the disjunction logical connector. MULTINUCLEAR-RESTATEMENT seems to occur as pairs of biconditional nuclei, although in principle there could be larger sets.

\section{$4 \quad$ Negating Relational Propositions}

The logical forms defined in Section 3 can be used to construct logical interpretations of a text. This is accomplished by restating the RST structure of the text as a nested relational proposition and mapping the predicates comprising the proposition to their respective logical forms. This suggests the possibility of analyzing texts as truth functional expressions. If so, we would expect that these expressions would be true or false, and that the determinants of their truth value would be indicative of the logical coherence of the discourse. This presupposes the ability to negate logical forms. And yet, based on the definitions presented in Section 3, it would seem that all relational propositions must be true. Presentational relational propositions are true because they are tautologies. Subject matter relational propositions are true because their satellites and nuclei are non-controversial. And while this observation - that relational propositions are by definition true-follows from the logical forms, and while these forms are in turn derived from their respective RST definitions, this clearly conflicts with both intuition and experience. RST analyzability is no guarantee of truth or logical coherence. As Mann and Thompson (1986b) and Marcu (1996) have shown, it is possible to assign plausible RST relations for incoherent discourse.

The apparent difficulty arises as a result of a disparity between the writer's intended effect and the actual effect on the reader. The RST relations are defined to indicate the writer's intended 
effect. This is consistent with theories of rhetoric in general (Heuboeck, 2009), but when reduced to its logical essence manifests itself as self-affirming reasoning, since it assumes that the effect has been achieved. Thus it is necessary to specify not only the forms of intended effect but their truth conditions as viewed from a skeptical perspective as well. The perspective of a skeptical reader can be used to identify potential points of failure within the logical forms. The potential points of failure can be used in constructing rules of negation for use in reasoning over logical forms.

The fundamental differences between presentational and subject matter relations require separate approaches for accomplishing this. The intended effect of presentational relations, to increase some inclination in the reader, is identified in the nucleus. If the writer is successful, i.e., if the reader accepts the effect, the reader accepts the situation as presented in the nucleus. The logical forms of the presentational relations build-in this acceptance. This is evident in the implicative structure of these relations. Presentational relational propositions are logically valid arguments. The premises of the argument are located in the left hand side (LHS) of the logical form, to the left of the outermost implication. From these premises, the right hand side (RHS) or conclusion, consisting of the nucleus, is deductively inferred. Thus the tautological structure of these relations presumes success for the writer's intended effect. While presenting the logical forms from the writer's perspective is consistent with the RST definitions, for the skeptical reader this amounts to begging the question. For the skeptical reader, it is the soundness of the LHS that is of interest. Although negation of the LHS will not necessarily negate the RHS, an LHS once negated provides no functional support for the intended effect. Since the burden of persuasion is on the LHS, its negation is sufficient for rejection of the RHS. Were this not the case, the relation would be subject matter rather than presentational. The LHS of a presentational relation consists of a major premise and a minor premise, as identified in Table 2. Denial of the LHS of the form can consist of the negation of either or both of the premises, or of the RHS itself.

\begin{tabular}{|l|l|l|l|}
\hline \multirow{2}{*}{\multicolumn{1}{|c|}{ Relation }} & \multicolumn{2}{c|}{ LHS } & \multicolumn{1}{c|}{ RHS } \\
\cline { 2 - 4 } & \multicolumn{1}{c|}{ Major Premise } & Minor Premise & Conclusion \\
\hline ANTITHESIS & $(s \vee n)$ & $\neg s$ & $n$ \\
\hline CONCESSION & $(\neg(s \rightarrow \neg n) \rightarrow n)$ & $\neg(s \rightarrow \neg n)$ & $n$ \\
\hline EVIDENCE, JUSTIFY, & $(s \rightarrow n)$ & $s$ & $n$ \\
MOTIVATION, ENABLEMENT, \\
BACKGROUND, PREPARATION, \\
RESTATEMENT, SUMMARY
\end{tabular}

Table 2. Presentational relations summary

The LHS of the ANTITHESIS relation is $((s \vee n) \wedge \neg s)$, which implies the RHS, $n$, such that ( $s$ $\vee n) \wedge \neg s) \rightarrow n$. The LHS includes several options for disputation by the skeptical reader. The negation of the LHS, $\neg((s \vee n) \wedge \neg s)$ follows whenever $s$ holds (the minor premise is negated), $n$ does not hold, or when the major premise is negated:

$$
(s \vee \neg n \vee \neg(s \vee n)) \rightarrow \neg((s \vee n) \wedge \neg s)
$$

For CONCESSION to succeed it is necessary that the satellite be compatible with the nucleus. The CONCESSION LHS is negated whenever the major or minor premise is negated, or when $n$ does not hold. Negating the minor premise challenges the compatibility between the satellite and the nucleus: 


$$
(\neg(\neg(s \rightarrow \neg n) \rightarrow n) \vee(s \rightarrow \neg n) \vee \neg n) \rightarrow \neg((\neg(s \rightarrow \neg n) \rightarrow n) \wedge \neg(s \rightarrow \neg n))
$$

Following the same approach, EVIDENCE is negated when the major or minor premise is negated, or when the nucleus is negated. Negating the major premise denies the implicative relationship between the satellite and the nucleus. Negating the minor premise $(\neg s)$ presents a valid argument for negation of the LHS. These same rules of negation also apply to the JUSTIFY, Motivation, Enablement, BACKground, Preparation, Restatement, and Summary relations:

$$
\neg(s \rightarrow n) \vee \neg s \vee \neg n \rightarrow \neg((s \rightarrow n) \wedge s)
$$

As defined in Section 3, several of the subject matter relations are subject matter implicatives. For some of these, the implication flows from satellite to nucleus (CAUSE, MEANS, Circumstance, SolutionhoOd), and for others the flow is from nucleus to satellite (Result, ELABORATION, EVALUATION, INTERPRETATION), but they all share the same general logical form, consisting of a conjunction of the satellite and nucleus and an implicative relation between the two. The negation rule for the $s \rightarrow n$ group is

$$
(\neg s \vee \neg n \vee \neg(s \rightarrow n)) \rightarrow \neg(s \wedge n \wedge(s \rightarrow n))
$$

and for the $n \rightarrow s$ group, the rule is

$$
(\neg s \vee \neg n \vee \neg(n \rightarrow s)) \rightarrow \neg(s \wedge n \wedge(n \rightarrow s))
$$

Since the logical forms of CONDITION and PURPOSE are material equivalence $(s \leftrightarrow n)$, negation requires that either the satellite be false while the nucleus is true, or the nucleus be false while the satellite is true:

$$
((s \wedge \neg n) \vee(\neg s \wedge n)) \rightarrow \neg(s \leftrightarrow n)
$$

UNCONDITIONAL asserts that the nucleus is not dependent on the satellite, in the sense that $n$, regardless of the value of $s$, or $(s \vee \neg s) \rightarrow n$. Negation of $n$ suffices to negate the relation:

$$
\neg n \rightarrow \neg((s \vee \neg S) \rightarrow n)
$$

OTHERWISE and UNLESS, both being exclusive disjunctions, are negated when either both the satellite and the nucleus are true or when both are false:

$$
((n \wedge s) \vee \neg(n \vee s)) \rightarrow \neg((n \vee s) \wedge \neg(n \wedge s))
$$

\section{The Logic of Relational Propositions}

The logical forms and rules of negation can be used in exploring the coherence of texts. More precisely, these forms and rules can be used to explore textual reasoning as reflected in an RST analysis. Any RST analysis can be restated as a nested relational proposition, and this nested relational proposition can be restated as a logical expression. As a simple example, the Music Day analysis from Mann and Thompson (1987b), shown in Figure 10, can be restated as this relational proposition: justify(concession(2,3), 1). The CONCESSION relation is the satellite of the JUSTIFY relation. Because the logical form of JUSTIFY is modus ponens $(((s \rightarrow n) \wedge s) \rightarrow n)$, the nested 
concession (2,3) appears twice, first as the conditional part of the major premise and again as the minor premise, as shown in Figure 11.

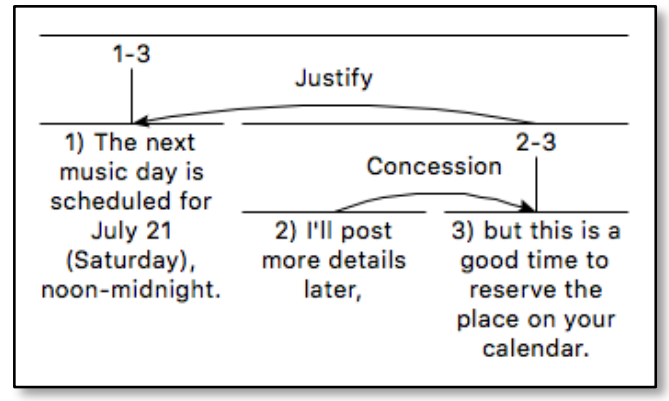

Figure 10. The Music Day analysis

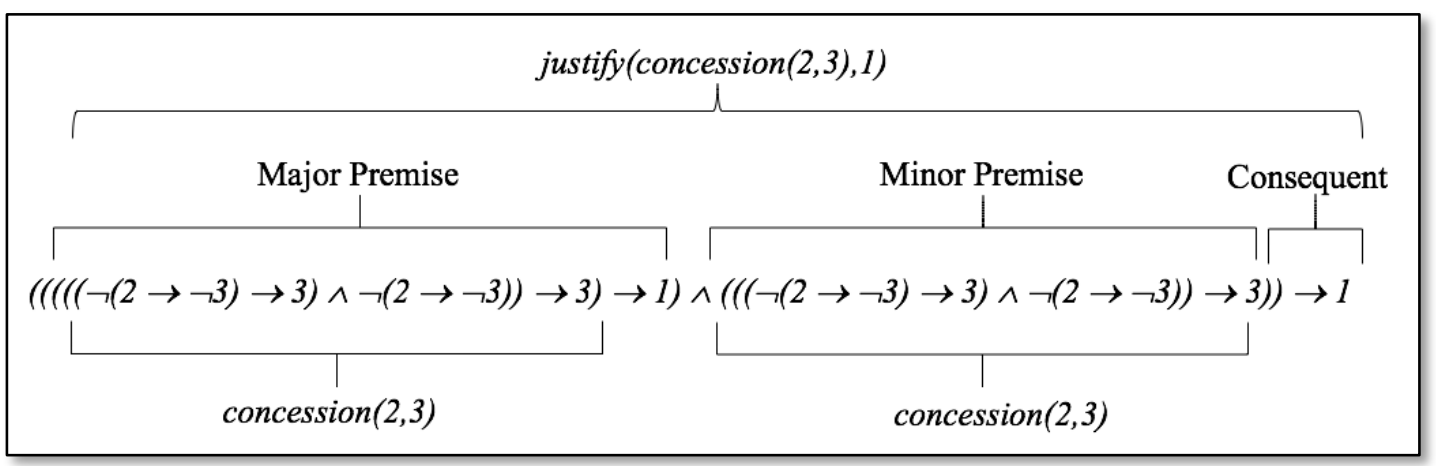

Figure 11. Music Day logic

Since both CONCESSION and JUSTIFY are presentational relations, for the purpose of this analysis, only the LHS of the forms are of interest. Here is the nested relational proposition reduced to the LHS of each relation:

$$
((((\neg(2 \rightarrow \neg 3) \rightarrow 3) \wedge \neg(2 \rightarrow \neg 3)) \rightarrow 3) \rightarrow 1) \wedge(((\neg(2 \rightarrow \neg 3) \rightarrow 3) \wedge \neg(2 \rightarrow \neg 3)) \rightarrow 3))
$$

To this we can selectively apply rules of negation. For example, if the reader challenges the CONCESSION relation by denying the compatibility between its nucleus and satellite, the result is negation of the entire expression:

$$
\begin{aligned}
& (2 \rightarrow \neg 3) \rightarrow \neg(((((\neg(2 \rightarrow \neg 3) \rightarrow 3) \wedge \neg(2 \rightarrow \neg 3)) \rightarrow 3) \rightarrow 1) \wedge(((\neg(2 \rightarrow \neg 3) \rightarrow 3) \wedge \\
& \neg(2 \rightarrow \neg 3)) \rightarrow 3))
\end{aligned}
$$

The negation is systemic because the negated CONCESSION is the minor premise of the JUSTIFY modus ponens argument. Within an RST analysis, when a rule of negation can be plausibly applied to a relational proposition, this is an indication of a potential misalignment either between the logic and the rhetorical coherence of the text or between the logic and the RST analysis. Thus the effects of faulty reasoning on the part of the writer and problematic RST encodings on the part of the analyst can be examined using the logic of relational propositions. The following presents a logical analysis of a more complex example. 
The RST analysis to be considered is "Bouquets in a Basket," shown in Figure 12. According to Mann and Thompson (1987a), the text is expository. The RST analysis consists primarily of subject matter relations. The text begins with the statement, There is a gardening revolution going on (1). Units 2-3 elaborate on the revolution mentioned in 1. The structure consisting of 1-3 is the satellite of a BACKGROUND relation. The nucleus of the BACKGROUND consists of a PURPOSE relation. Mann and Thompson mention that unit 4, the satellite of a PURPOSE relation, presents a possible goal, of creating "your own" bouquet, with segments 5-8 providing the method.

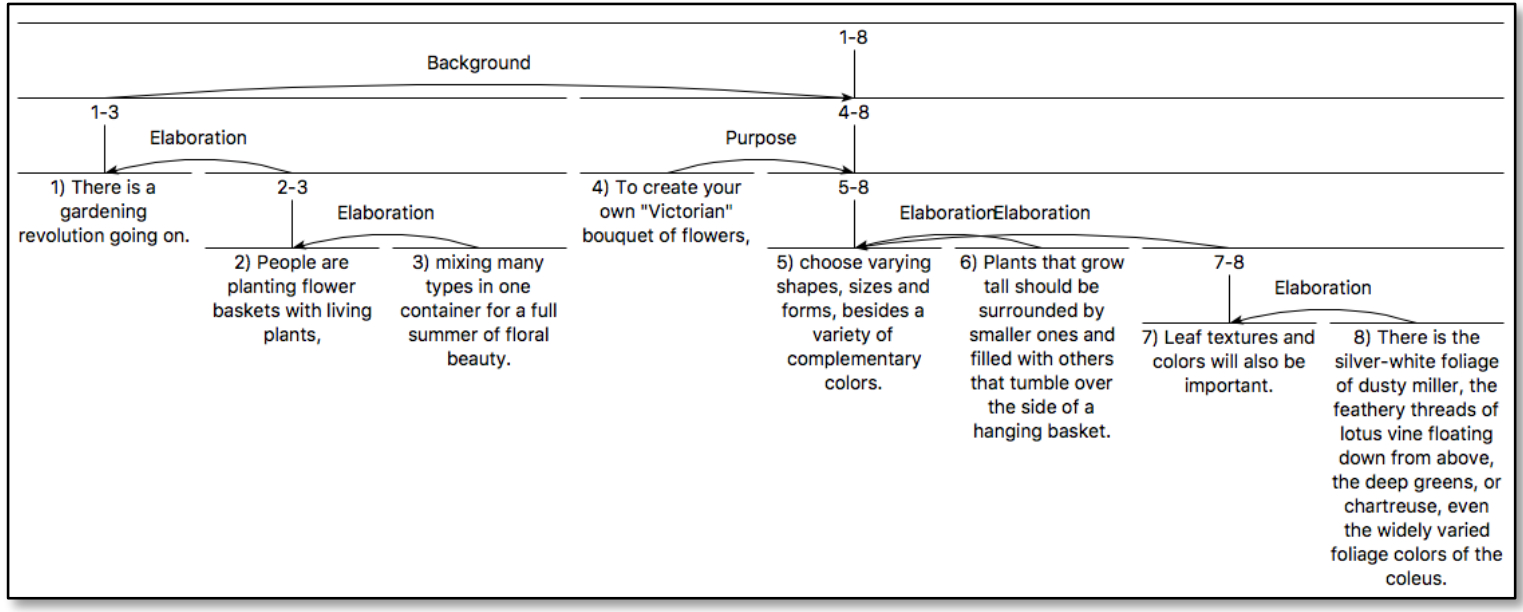

Figure 12. RST Analysis of the Bouquets in a Basket text

The logic of the text, as implicit in this RST analysis, may be summarized as follows: As the nucleus of an ELABORATION relation, the statement that there is a gardening revolution going on (1) is the antecedent of an implicative relation with units 2-3. By definition of ELABORATION, that people are planting flower baskets with living plants, and mixing many types in one container for a full summer of floral beauty follows from the assertion that there is a gardening revolution going on:

$$
(1 \wedge(2 \wedge 3 \wedge(2 \rightarrow 3)) \wedge(1 \rightarrow(2 \wedge 3 \wedge(2 \rightarrow 3))))
$$

By definition of BACKGROUND, from 1-3, it follows that the reader may create a bouquet of flowers (4) using the method presented in 5-8. And by definition of PURPOSE, the method presented in text span $5-8$ is materially equivalent with 4 :

$$
(((5 \wedge 6 \wedge(5 \rightarrow 6)) \wedge(5 \wedge(7 \wedge 8 \wedge(7 \rightarrow 8)) \wedge(5 \rightarrow(7 \wedge 8 \wedge(7 \rightarrow 8))))) \leftrightarrow 4)
$$

Since BACKGROUND is a presentational relation, and it is an overarching relation for this text, the logical expression representing the text is this tautology:

$$
\begin{aligned}
& (((1 \wedge(2 \wedge 3 \wedge(2 \rightarrow 3)) \wedge(1 \rightarrow(2 \wedge 3 \wedge(2 \rightarrow 3)))) \rightarrow(((5 \wedge(7 \wedge 8 \wedge(7 \rightarrow 8)) \wedge(5 \rightarrow \\
& (7 \wedge 8 \wedge(7 \rightarrow 8))) \wedge(5 \wedge 6 \wedge(5 \rightarrow 6))) \leftrightarrow 4)) \wedge(1 \wedge(2 \wedge 3 \wedge(2 \rightarrow 3)) \wedge(1 \rightarrow(2 \wedge 3 \wedge \\
& (2 \rightarrow 3))))) \rightarrow(((5 \wedge(7 \wedge 8 \wedge(7 \rightarrow 8)) \wedge(5 \rightarrow(7 \wedge 8 \wedge(7 \rightarrow 8)))) \wedge(5 \wedge 6 \wedge(5 \rightarrow 6))) \\
& \leftrightarrow 4))
\end{aligned}
$$

Thus, according to the RST analysis, the text is logically coherent. This would be expected of any RST analysis, since the logical forms arise from the RST definitions. The logic becomes more 
interesting when the rules of negation are applied. There are a few inferences within this RST analysis that may seem problematic to the skeptical reader. First, as a subject matter relation, ELABORATION assumes that the reader will accept both the satellite and nucleus. If either is questionable, some other relation must be applied. Taken on its own, there is a gardening revolution going on (1) will seem puzzling to readers if the hyperbolic use of the term revolution is not obvious. In that case, substantiation would be needed. If for lack of substantiation the statement is denied, the consequences are systemic, and the LHS of the BACKGROUND relation is negated, resulting in this tautology:

$$
\begin{aligned}
& (\neg 1 \rightarrow \neg((1 \wedge(2 \wedge 3 \wedge(2 \rightarrow 3)) \wedge(1 \rightarrow(2 \wedge 3 \wedge(2 \rightarrow 3)))) \rightarrow(((5 \wedge(7 \wedge 8 \wedge(7 \rightarrow 8)) \\
& \wedge(5 \rightarrow(7 \wedge 8 \wedge(7 \rightarrow 8)))) \wedge(5 \wedge 6 \wedge(5 \rightarrow 6))) \leftrightarrow 4)) \wedge(1 \wedge(2 \wedge 3 \wedge(2 \rightarrow 3)) \wedge(1 \rightarrow(2 \\
& \wedge 3 \wedge(2 \rightarrow 3))))))
\end{aligned}
$$

However, the lack of substantiation is not within the text, but emerges only when accessing the logic of the text through the intermediation of the RST analysis. That is, the logic of the ELABORATION relation is inconsistent with the logical coherence of the text. The satellite, rather than following from the nucleus, is needed to give credence to the claim asserted by the nucleus. This can be satisfied using EVIDENCE rather than ELABORATION for the relation between 1 and 2 .

A second problematic inference is found in the PURPOSE relation. It is significant that the satellite of this relation, segment 4, addresses the reader directly: ...create your own "Victorian" bouquet of flowers. Without some indication of why and how the reader should carry out such an activity, the BACKGROUND relation provides no support other than comprehension. Moreover, the nucleus of the BACKGROUND is not 4, but the PURPOSE relation itself, containing the structure of nested ELABORATION relations (5-8) for which 4 is satellite. The PURPOSE of 5-8, to create your own "Victorian" bouquet of flowers, must stand on its own. This places 4 at risk of negation. If the reader is disinclined to create a bouquet and denies 4, the PURPOSE relation fails. Negation of the nucleus implies negation of the satellite, and this results in negation of the BACKGROUND relation.

Concerns such as these are opportunities for assessing the structure of the text. An alternative analysis, one that would align the logic of the text and its RST structure, would be to code segment 4 as the nucleus of a Motivation/EnABLEMENT schema, as shown in Figure 13. Segments 1-3 are intended not merely to impart comprehension of what follows, but to increase the reader's desire to perform the action, i.e., to create a bouquet. By offering the reader the prospect of a full summer of floral beauty, segments 1-3 are intended to increase the reader's desire to create a basket, and segments 5-8 increase the reader's ability to do so, by providing a method involving choices of shapes, sizes, forms, and colors of the flowers, height of the plants, leaf textures, and leaf colors. With this revised analysis, segment 4 emerges as the locus of effect for the text, and as the logical consequent as well. Without alignment between RST structure with the logic of relational propositions, the inferences indicated by the original RST analysis lack plausibility and are subject to negation. By aligning the RST structure with its logical inferences, the organization of the text comes into focus: segment 1 follows from text span 2-3, and segment 4 follows from 1-3, representing the MOTIVATION part of the text. Conjunctively, 4 also follows from 5-6, representing the ENABLEMENT part of the text. Here is the Motivation conjunct:

$$
\begin{aligned}
& (((((((2 \wedge 3 \wedge(2 \rightarrow 3)) \rightarrow 1) \wedge(2 \wedge 3 \wedge(2 \rightarrow 3))) \rightarrow 1) \rightarrow 4) \wedge((((2 \wedge 3 \wedge(2 \rightarrow 3)) \rightarrow 1) \\
& \wedge(2 \wedge 3 \wedge(2 \rightarrow 3))) \rightarrow 1)) \rightarrow 4)
\end{aligned}
$$

And this is the ENABLEMENT conjunct: 


$$
\begin{aligned}
& ((((5 \wedge 6 \wedge(5 \rightarrow 6)) \wedge(5 \wedge(7 \wedge 8 \wedge(7 \rightarrow 8)) \wedge(5 \rightarrow(7 \wedge 8 \wedge(7 \rightarrow 8))))) \rightarrow 4) \wedge((5 \wedge 6 \\
& \wedge(5 \rightarrow 6)) \wedge(5 \wedge(7 \wedge 8 \wedge(7 \rightarrow 8)) \wedge(5 \rightarrow(7 \wedge 8 \wedge(7 \rightarrow 8)))))) \rightarrow 4)
\end{aligned}
$$

In their commentary on this analysis, Mann and Thompson seemed to have recognized this, as indicated by their observation that 4 presents the possible goal, and that segments 5-8 provide the method. But they did not choose to code the text as such. An RST analysis that cannot pass the test of logic is an analysis in need of attention. As demonstrated here, the revisions indicated by the logic of relational propositions need not be merely a matter of tweaking a few relations. It may provide an alternative perspective on the text. In this example, the difference is between expository and argumentative. That the logic of relational propositions can be used to assess an existing RST analysis shows that it can also be used in the development of new analyses as well. The decision process would be the same: for any structure under consideration, identify the corresponding logical expressions and their consequences. If these lead to inconsistencies, either the text is problematic or the structure is in need of review.

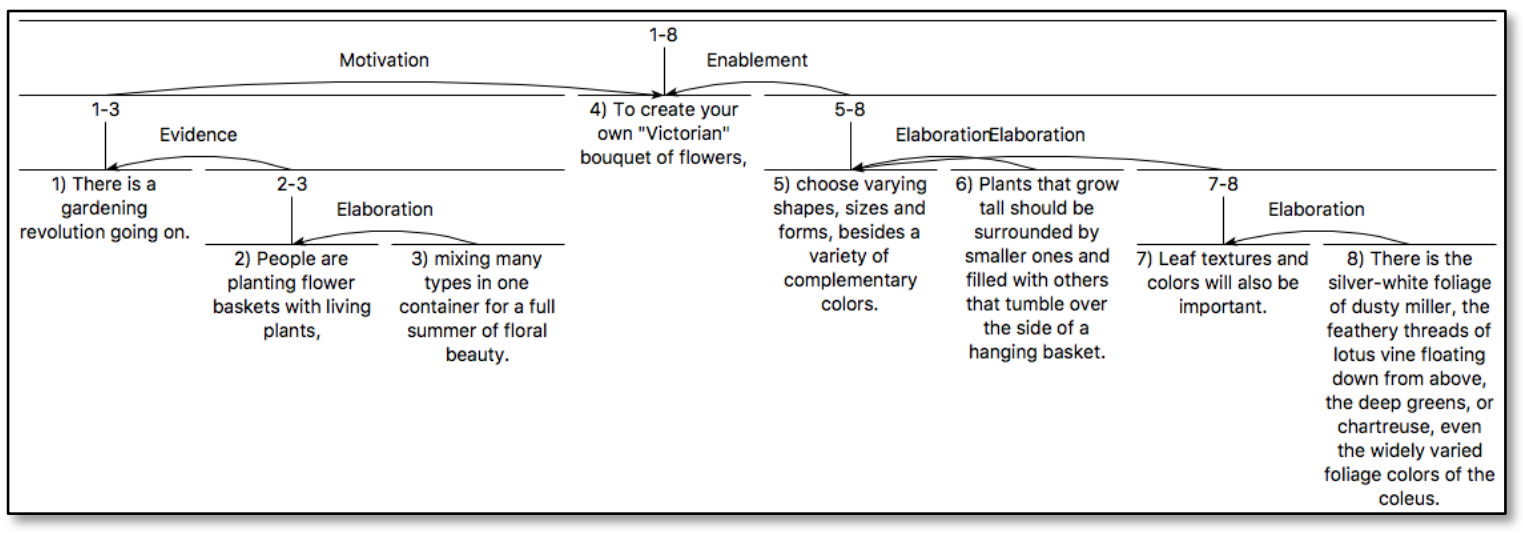

Figure 13. Alternative of analysis of Bouquets in a Basket

\section{Conclusion}

The relationship between coherence relations and logic has been an active area of research at least since Hobbs $(1979,1985)$ used logic to define a set of relations for use in an automated inference system. Using this approach, relations could be represented in a manner akin to (but not identical with) the predicate calculus (Hobbs, 1979). There have been numerous other studies in the logic of coherence relations (e.g., Danlos, 2008; González \& Ribas, 2008; Groenendijk, 2009; Marcu, 2000; Sanders et al., 1992; Wong, 1986). Much of the recent research in this area has been influenced by the work of Asher and Lascarides (2003), with their development of Segmented Discourse Representation Theory (SDRT). SDRT is based in part on Discourse Representation Theory (DRT), a theory of dynamic semantics that attempts to account for the context dependence of meaning, based on the observation that how a sentence is interpreted depends on what has occurred previously within the discourse (Kamp \& Reyle, 1993; Kamp, Van Genabith, \& Reyle, 2011). That is, the discourse context is dynamically modified with each successive utterance, and this updated context informs the interpretation of whatever comes next. Building on DRT, SDRT uses dynamic semantics to generate interpretations of discourse. Updates to the interpretation are made using a glue logic which identifies appropriate rhetorical relations for linking new information to old, assigning a compositional and dynamic semantic interpretation for the discourse. The aims of this paper have been more modest than those of SDRT. In this paper I have demonstrated the use of relational propositions as a means for investigating texts as expressions in propositional logic. While SDRT defines a set of rules for deriving rhetorical structures from 
logical forms, the logic of relational propositions provides a set of rules for deriving logical forms from rhetorical structures. While SDRT is a theory of dynamic discourse interpretation, the logic of relational propositions is based on a static logical interpretation of intended effect. Thus, the two theories are each motivated by rather different goals, with rather different results.

This paper has shown that any text analyzable using RST can be restated as an expression in propositional logic. Relational propositions play a key role in this. By providing a theoretical basis for treating RST structures as assertions, relational propositions provide a bridge between RST analyses and propositional logic. In order to apply the logic of relational propositions to complete texts, rather than individual relations, it was necessary to extend the theory, so that any multiunit RST span can be restated as a nested relational proposition. For each of the RST relations there is a defined logical form reflecting the logical relationship between the satellite and the nucleus. Within a nested relational proposition, the forms may be substituted for each of the constituent relations. Mapping the forms into a nested relational proposition yields a logical expression of the text. The reduction of texts to logical forms reveals that logical inference is pervasive within coherent text. The paper has also defined a set of rules for negation of logical forms. The logical forms and rules of negation can be used to examine the logical coherence of texts. The status of the logical coherence of a text can be used to support RST analysis. Because there is a correspondence between logical coherence and the functional relationships of RST, an RST analysis that cannot pass the test of logic is indicative either of a problematic analysis or textual incoherence.

There are several potential applications of this research. If integrated with computational methods for generating RST analyses (e.g., Corston-Oliver, 1998; Hernault, Prendinger, duVerle, \& Ishizuka, 2010; Pardo, Nunes, \& Rino, 2004; Soricut \& Marcu, 2003), the method presented here could lead to useful tools for scalable analysis of large text collections. Some other potential uses include contributions to knowledge representation, automated reasoning, controlled natural languages, cross-document analysis (Cardoso, Jorge, \& Pardo, 2015; Radev, 2000), and integration with research in semantic equivalence, entailment, and knowledge extraction (Androutsopoulos \& Malakasiotis, 2010; Gangemi, 2013; Zhang \& Patrick, 2005). Some areas for future study include development of a computational framework for the study of the logic of RST analyses, studies in the interrelationship between logic and rhetoric, a more in-depth look at multinuclear relations, and investigation of various genres of discourse using the methods described in this paper.

\section{References}

Abelen, E., Redeker, G., \& Thompson, S. (1993). The rhetorical structure of US-American and Dutch fund-raising letters. Text, 3, 323-350.

Androutsopoulos, I., \& Malakasiotis, P. (2010). A survey of paraphrasing and textual entailment methods. Journal of Artificial Intelligence Research, 38, 135-187.

Asher, N., \& Lascarides, A. (2003). Logics of conversation. Cambridge, UK: Cambridge University Press.

Azar, M. (1999). Argumentative text as rhetorical structure: An application of rhetorical structure theory. Argumentation, 13(1), 97-114.

Boole, G. (1854). An investigation of the laws of thought on which are founded the mathematical theories of logic and probabilities (Dover 1958 reprint ed.). New York: Macmillan.

Brooks, A. W. (2013). Get excited: Reappraising pre-performance anxiety as excitement. Journal of Experimental Psychology, 143(3), 1144-1158.

Cardoso, P. C. F., Jorge, M. L. R. C., \& Pardo, T. A. S. (2015). Exploring the Rhetorical Structure Theory for multi-document summarization Congreso de la Sociedad Española para el Procesamiento del Lenguaje Natural (Vol. XXXI). Alicante, Spain: Sociedad Española para el Procesamiento del Lenguaje Natural. 
Carlson, L., \& Marcu, D. (2001, September). Discourse tagging reference manual. Retrieved from ftp://ftp.isi.edu/isi-pubs/tr-545.pdf

Copi, I. M. (1967). Symbolic logic. New York: Macmillan.

Corston-Oliver, S. H. (1998). Computing representations of the structure of written discourse. (dissertation), University of California, Santa Barbara, CA.

Danlos, L. (2008). Strong generative capacity of RST, SDRT and discourse dependency DAGSs. In A. Benz \& P. Kühnlein (Eds.), Constraints in discourse (pp. 69-95). Amsterdam: Benjamins.

Forbus, K. D. (2016). Software social organisms: Implications for measuring AI progress. AI Magazine, 37(1), 85-90.

Gangemi, A. (2013). A Comparison of knowledge extraction tools for the Semantic Web. In P. Cimiano, O. Corcho, V. Presutti, L. Hollink, \& S. Rudolph (Eds.), The Semantic Web: Semantics and Big Data (pp. 351-366). Berlin, Heidelberg: Springer.

González, M., \& Ribas, M. (2008). The construction of epistemic space via causal connectives. In I. Kecskes \& J. Mey (Eds.), Intention, common ground and the egocentric speaker-hearer (pp. 127-149). Berlin: de Gruyter.

Groenendijk, J. (2009). Inquisitive semantics: Two possibilities for disjunction. In P. Bosch, D. Gabelaia, \& J. Lang (Eds.), Logic, language, and computation (pp. 80-94). Berlin, Heidelberg: Springer Berlin Heidelberg.

Grosz, B., \& Sidner, C. (1986). Attention, intentions, and the structure of discourse. Computational Linguistics, 12(3), 175-204.

Halliday, M. A. K., \& Hasan, R. (1976). Cohesion in English. London: Longman.

Hernault, H., Prendinger, H., duVerle, D. A., \& Ishizuka, M. (2010). HILDA: A Discourse parser using Support Vector Machine classification. Dialogue and Discourse, 1(3), 1-33.

Heuboeck, A. (2009). Some aspects of coherence, genre and rhetorical structure - and their integration in a generic model of text. Language Studies Working Papers, 1, 35-45.

Hintikka, J. (2007). Socratic epistemology: Explorations of knowledge-seeking by questioning. New York: Cambridge University Press.

Hobbs, J. R. (1979). Coherence and coreference. Cognitive Science, 3, 67-90.

Hobbs, J. R. (1985). On the coherence and structure of discourse (CSLI-85-37). Stanford, CA: Center for the Study of Language and Information, Stanford University. Retrieved from http://www.isi.edu/ hobbs/ocsd.pdf

Hovy, E. H. (1990). Parsimonious and profligate approaches to the question of discourse structure relations. Proceedings of the Fifth International Workshop on Natural Language Generation. Pittsburgh, PA: Association for Computational Linguistics.

Hovy, E. H., \& Maier, E. (1993). Parsimonious or profligate: How many and which discourse relations? Marina del Rey, CA: Information Sciences Institute, University of Southern California.

Iruskieta, M. (2014). A description of pragmatics rhetorical structure and its evaluation in computational linguistics. Paper presented at the Programa de Pós-Graduação em Letras, Maringa, Brazil.

Kamp, H., \& Reyle, U. (1993). From discourse to logic: Introduction to model-theoretic semantics of natural language, formal logic and Discourse Representation Theory. Dordrecht: Kluwer.

Kamp, H., Van Genabith, J., \& Reyle, U. (2011). Discourse representation theory. In D. Gabbay \& F. Guenthner (Eds.), Handbook of philosophical logic, Volume 15 (2nd ed., pp. 125-294). Dordrecht: Springer.

Knott, A., Oberlander, J., O'Donnell, M., \& Mellish, C. (2001). Beyond elaboration: The interaction of relations and focus in coherent text. In T. Sanders, J. Schilperoord, \& W. Spooren (Eds.), 
Text Representation: Linguistic and Psycholinguistic Aspects (pp. 181-196). Amsterdam: John Benjamins.

Kolodny, N. (2007). How does coherence matter? Proceedings of the Aristotelian Society (Vol. 107, pp. 229-263). Oxford: Oxford University Press.

Mann, W. C., \& Matthiessen, C. M. I. M. (1990). Functions of language in two frameworks. Marina del Rey, CA: Information Sciences Institute.

Mann, W. C., \& Thompson, S. A. (1985). Assertions from discourse structure (Technical Report No. ISI/RS-85-155). Marina del Rey, California: Information Sciences Institute.

Mann, W. C., \& Thompson, S. A. (1986a). Assertions from discourse structure. HLT '86: Proceedings of the workshop on strategic computing natural language (pp. 257-270). Morristown, NJ: Association for Computational Linguistics.

Mann, W. C., \& Thompson, S. A. (1986b). Relational propositions in discourse. Discourse Processes, 9(1), 57-90.

Mann, W. C., \& Thompson, S. A. (1987a). Rhetorical Structure Theory: A framework for the analysis of texts. IPRA Papers in Pragmatics, 1, 1-21.

Mann, W. C., \& Thompson, S. A. (1987b). Rhetorical structure theory: A theory of text organization (ISI/RS-87-190). Marina del Rey, CA: University of Southern California, Information Sciences Institute (ISI).

Mann, W. C., \& Thompson, S. A. (1988). Rhetorical structure theory: Towards a functional theory of text organization. Text, 8(3), 243-281.

Mann, W. C., \& Thompson, S. A. (2000a). Toward a theory of reading between the lines: An exploration in discourse structure and implicit communication. Paper presented at the Seventh International Pragmatics Conference, Budapest, Hungary.

Mann, W. C., \& Thompson, S. A. (2000b). Two views of rhetorical structure theory. Proceedings of the 10th Annual Meeting of the Society for Text and Discourse. Lyon, France.

Marcu, D. (1996). Distinguishing between coherent and incoherent texts. The Proceedings of the Student Conference on Computational Linguistics in Montreal (pp. 136-143).

Marcu, D. (2000). The theory and practice of discourse parsing and summarization. Cambridge, MA: MIT Press.

Nicholas, N. (1994). Problems in the application of rhetorical structure theory to text generation. (masters thesis), University of Melbourne, Melbourne, Australia.

Pardo, T. A. S., Nunes, M. d. G. V., \& Rino, L. H. M. (2004). DiZer: An automatic discourse analyzer for Brazilian Portuguese. Advances in Artificial Intelligence - SBIA 2004 17th Brazilian Symposium on Artificial Intelligence, Sao Luis, Maranhao, Brazil, September 29Ocotber 1, 2004. Proceedings. Berlin: Springer.

Radev, D. R. (2000). A common theory of information fusion from multiple text sources step one: cross-document structure Proceedings of the 1st SIGdial workshop on Discourse and dialogue - Volume 10 (pp. 74-83). Hong Kong: Association for Computational Linguistics.

Rubin, V. L., \& Vashchilko, T. (2012). Identification of truth and deception in text: Application of vector space model to rhetorical structure theory Proceedings of the Workshop on Computational Approaches to Deception Detection (pp. 97-106). Avignon, France: Association for Computational Linguistics.

Russell, B. (1906). The theory of implication. American Journal of Mathematics, 28(2), 159-202

Sanders, T. J. M., Spooren, W. P. M., \& Noordman, L. G. M. (1992). Toward a taxonomy of coherence relations. Discourse Processes, 15, 1-35.

Soricut, R., \& Marcu, D. (2003). Sentence level discourse parsing using syntactic and lexical information. Proceedings of the 2003 Conference of the North American Chapter of the Association for Computational Linguistics on Human Language Technology - Volume 1 (pp. 149-156). Edmonton, Canada: Association for Computational Linguistics. 
Sowa, J. F. (2000). Knowledge representation: Logical, philosophical, and computational foundations. Pacific Grove, CA: Brooks/Cole.

Stede, M. (2008). Disambiguating rhetorical structure. Research on Language and Computation, 6(3), 311-332.

Stede, M., Taboada, M., \& Das, D. (2017). Annotation guidelines for rhetorical structure. University of Potsdam and Simon Fraser University. Retrieved from http://www.sfu.ca/ mtaboada/docs/research/RST_Annotation_Guidelines.pdf

Stent, A. J. (2000). Rhetorical structure in dialog. Paper presented at the 2nd International Natural Language Generation Conference (INLG'2000), Patras, Greece.

Taboada, M. (2006). Discourse markers as signals (or not) of rhetorical relations. Journal of Pragmatics, 38(4), 567-592.

Taboada, M., \& Mann, W. C. (2006a). Applications of rhetorical structure theory. Discourse Studies, 8(4), 567-588.

Taboada, M., \& Mann, W. C. (2006b). Rhetorical structure theory: Looking back and moving ahead. Discourse Studies, 8(3), 423-459.

Taboada, M., \& Renkema, J. (2008). Discourse relations reference corpus: RST analyses of 65 texts. Burnaby, Tilburg: Simon Fraser University and Tilburg University. Retrieved from http://www.sfu.ca/rst/06tools/discourse_relations_corpus.html

Thagard, P. (1989). Extending explanatory coherence. Behavioral and Brain Sciences, 12, 435502.

Thompson, S. A., \& Mann, W. C. (1986). A discourse view of concession in written English. Proceedings of the Second Annual Meeting of the Pacific Linguistics Conference (pp. 435447). Eugene, Oregon: University of Oregon, Eugene.

Thompson, S. A., \& Mann, W. C. (1987). Antithesis: A study in clause combining and discourse structure. In R. Steele \& T. Threadgold (Eds.), Language Topics: Essays in Honour of Michael Halliday, Volume II (pp. 359-381). Amsterdam: John Benjamins.

Whitehead, A. N., \& Russell, B. (1910). Principia mathematica (Vol. 1). Cambridge, England: Cambridge University Press.

Wong, W.-K. C. (1986). A theory of argument coherence (TR86-29). Austin, Texas: Artificial Intelligence Laboratory.

Zhang, Y., \& Patrick, J. (2005). Paraphrase identification by text canonicalization. Proceedings of the Australasian Language Technology Workshop (pp. 160-166). Sydney, Australia. 